\begin{tabular}{|c|l|}
\hline Title & Formulas for q-spherical functions using inverse scattering theory of reflectionless Jacobi operators \\
\hline Author(s) & Diejen, J. F. V an; Kirillov, A. N \\
\hline Citation & Hokkaido University Preprint Series in Mathematics, 430, 1-33 \\
\hline Issue Date & 1998-9-1 \\
\hline DOI & 10.14943/83576 \\
\hline Doc URL & http://hdl.handle.net/2115/69180 \\
\hline Type & bulletin (article) \\
\hline File Information & pre430.pdf \\
\hline
\end{tabular}

Instructions for use 


\section{FORMULAS FOR $q$-SPHERICAL FUNCTIONS USING INVERSE SCATTERING THEORY OF REFLECTIONLESS JACOBI OPERATORS}

J. F. Van Diejen and A. N. Kirillov

Series \#430. September 1998 


\section{HOKKAIDO UNIVERSITY \\ PREPRINT SERIES IN MATHEMATICS}

\#405 K. Yokoyama, Global existence of classical solutions to systems of wave equations with critical nonlinearity in three space dimensions, 25 pages. 1998.

\#406 F. Hiroshima, Ground states and spectrum of quantum electrodynamics of non-relativistic particles, 58 pages. 1998.

\#407 N. Kawazumi and T. Uemura, Riemann-Hurwitz formula for Morita-Mumford classes and surface symmetries, 9 pages. 1998.

\#408 T. Nakazi and K. Okubo, Generalized Numerical Radius And Unitary $\rho$-Dilation, 12 pages. 1998.

\#409 Y. Giga and K. Ito, Loss of convexity of simple closed curves moved by surface diffusion, 16 pages. 1998.

\#410 Y. Giga, K. Inui and S. Matsui, On the Cauchy problem for the Navier-Stokes equations with nondecaying initial data, 34 pages. 1998.

\#411 S. Izumiya, D. Pei and T. Sano, The lightcone gauss map and the lightcone developable of a spacelike curve in Minkowski 3-space, 16 pages. 1998.

\#412 M. Tsujii, Absolutely continuous invarient measures for piecewise real-analytic expanding maps on the plane, 18 pages. 1998.

\#413 J.-P. Brasselet, D. Lehmann, J. Seade and T. Suwa, Milnor classes of local complete intersections, 40 pages. 1998.

\#414 T. Suwa, Dual class of a subvariety, 19 pages. 1998.

\#415 M. Tsujii, Piecewise expanding maps on the plane with singular ergodic properties, 9 pages. 1998.

\#416 T. Yoshida, Categorical aspects of generating functions( I ): Exponential formulas and Krull-Schmidt categories, 44 pages. 1998.

\#417 K. Yamaguchi, $G_{2}$-Geometry of overdetermined systems of second order, 22 pages. 1998.

\#418 M. Ishikawa and S. Matsui, Existence of a forward self-similar stagnation flow of the Navier-Stokes equations, 8 pages. 1998.

\#419 S. Izumiya, H. Katsumi and T. Yamasaki, The rectifying developable and the spherical Darboux image of a space curve, 16 pages. 1998.

\#420 R. Kobayashi and Y. Giga, Equations with singular diffusivity, 45 pages. 1998.

\#421 D. Pei and T. Sano, The focal developable and the binormal indicatrix of a nonlightlike curve in Minkowski 3-space, 14 pages. 1998.

\#422 R. Kobayashi, J. A. Warren and W. C. Carter, Modeling grain boundaries using a phase field technique, 12 pages. 1998.

\#423 T. Tsukada, Reticular Legendrian Singularities, 28 pages. 1998.

\#424 A. N. Kirillov and M. Shimozono, A generalization of the Kostka-Foulkes polynomials, 37 pages. 1998.

\#425 M. Nakamura, Strichartz estimates for wave equations in the homogeneous Besov space, 17 pages. 1998.

\#426 A. Arai, On the essential spectra of quantum field Hamiltonians, 18 pages. 1998.

\#427 T. Sano, Bifurcations of affine invariants for one parameter family of generic convex plane curves, 11 pages. 1998.

\#428 F. Hiroshima, Ground states of a model in quantum electrodynamics, 48 pages. 1998.

\#429 F. Hiroshima, Uniqueness of the ground state of a model in quantum electrodynamics: A functional integral approach, 32 pages. 1998. 


\title{
FORMULAS FOR $q$-SPHERICAL FUNCTIONS USING INVERSE SCATTERING THEORY OF REFLECTIONLESS JACOBI OPERATORS
}

\author{
J.F. VAN DIEJEN AND A.N. KIRILLOV
}

\begin{abstract}
We study the spectral problem associated to a Ruijsenaars-type $(q$-)difference version of the one-dimensional Schrödinger operator with PöschlTeller potential. The eigenfunctions are constructed explicitly with the aid of the inverse scattering theory of reflectionless Jacobi operators. As a result, we arrive at combinatorial formulas for basic hypergeometric deformations of zonal spherical functions on odd-dimensional hyperboloids and spheres.
\end{abstract}

\section{Contents}

1. Introduction

2. A generalized Sato formula for finite-difference operators 4

3. Combinatorics of the difference Pöschl-Teller problem 7

4. Basic hypergeometry 11

4.1. A basic hypergeometric representation 11

4.2. Bispectrality 14

4.3. Relation to Ruijsenaars' wave functions 16

5. $q$-Spherical functions $\quad 17$

5.1. Combinatorial formulas 17

$\begin{array}{ll}\text { 5.2. Regularity } & 19\end{array}$

5.3. $q$-Ultraspherical polynomials $\quad 20$

5.4. Zonal spherical functions 22

Appendix A. Reflectionless bidiagonal Jacobi operators and the discrete Sato formula 24

A.1. Spectral data 25

A.2. The discrete Gelfand-Levitan-Marchenko equation 25

A.3. Inverse scattering of reflectionless bidiagonal Jacobi operators $\quad 26$

A.4. The discrete Sato formula 28

References $\quad 30$

Date: September 1998.

1991 Mathematics Subject Classification. Primary: 33D55; Secondary: 33D80, 43A90, 81U40.

The research of JFvD was supported in part by the Fondo Nacional de Desarrollo Cientifico y Tecnologico (FONDECYT) through Grant \# 1980832. 


\section{INTRODUCTION}

This paper investigates the explicit structure of the eigenfunctions of a onedimensional Ruijsenaars-type difference operator of the form [Ru1, Ru2]

$$
\mathcal{D}=v_{+}^{1 / 2}(x) e^{\beta \frac{\mathrm{d}}{\mathrm{d} x}} v_{-}^{1 / 2}(x)+v_{-}^{1 / 2}(x) e^{-\beta \frac{\mathrm{d}}{\mathrm{d} x}} v_{+}^{1 / 2}(x), \quad x \in \mathbb{R},
$$

where $\left(e^{ \pm \beta \frac{\mathrm{d}}{\mathrm{dx}}} \psi\right)(x)=\psi(x \pm \beta)$ for $\psi: \mathbb{R} \rightarrow \mathbb{C}$ and

$$
\begin{aligned}
v_{ \pm}(x) & =\frac{\cosh \alpha(x \pm \beta g)}{\cosh (\alpha x)} \\
& =q^{\mp \beta g / 2}\left(\frac{1+q^{x \pm \beta g}}{1+q^{x}}\right), \quad q=e^{-2 \alpha} .
\end{aligned}
$$

This difference operator may be looked upon as a finite-difference version of the one-dimensional Schrödinger operator with Pöschl-Teller potential [Flu, Ti]. More concretely, the relevant Schrödinger operator is recovered via a formal expansion in the step size parameter $\beta$ :

$$
\mathcal{D}=2+\beta^{2} \mathcal{D}_{0}+o\left(\beta^{3}\right)
$$

with

$$
\mathcal{D}_{0}=\frac{\mathrm{d}^{2}}{\mathrm{~d} x^{2}}+\frac{g(g-1) \alpha^{2}}{\cosh ^{2}(\alpha x)}
$$

Hence, one has that (formally) $\mathcal{D}_{0}=\lim _{\beta \rightarrow 0}(\mathcal{D}-2) / \beta^{2}$. In the above formulas $\alpha$ and $\beta$ are scaling parameters (positive say) and $g$ represents a (nonnegative) coupling parameter. One may actually absorb the step size parameter $\beta$ into $\alpha$ by means of the rescaling $x \rightarrow \beta x$. From now on we shall therefore set the step size $\beta$ equal to 1 without loss of generality. In addition, we shall also assume that $g$ is a positive integer: $g=N+1, N \in \mathbb{N}$. The latter assumption guarantees, roughly speaking, that our Ruijsenaars-type difference Pöschl-Teller operator is reflectionless.

Restriction of the difference Pöschl-Teller operator to functions with support in the integer lattice $\mathbb{Z}$ gives rise to a Jacobi operator of the form [SZ]

$$
D=a_{n} T+a_{n-1} T^{-1}, \quad n \in \mathbb{Z},
$$

where $(T \psi)(n)=\psi(n+1)$ for $\psi: \mathbb{Z} \rightarrow \mathbb{C}$ and

$$
\begin{aligned}
a_{n} & =\left(\frac{\cosh \alpha(n+N+1) \cosh \alpha(n-N)}{\cosh \alpha(n+1) \cosh (\alpha n)}\right)^{1 / 2} \\
& =\frac{\left(1+q^{n+N+1}\right)^{1 / 2}\left(1+q^{n-N}\right)^{1 / 2}}{\left(1+q^{n+1}\right)^{1 / 2}\left(1+q^{n}\right)^{1 / 2}}, \quad q=e^{-2 \alpha} .
\end{aligned}
$$

That is to say, formally the Jacobi operator $D(1.3 \mathrm{a}),(1.3 \mathrm{~b})$ is obtained from the difference operator $\mathcal{D}$ (1.1a), (1.1b) (with $\beta=1$ and $g=N+1$ ) by setting $x=n$ and commuting all coefficients to the left. The diagonal of the Jacobi operator under consideration is zero; as a consequence, we are dealing with the special situation of a Jacobi operator with a bidiagonal rather than a tridiagonal structure (as usual). Our Jacobi operator should be thought of as a self-adjoint discrete difference operator in the Hilbert space $l^{2}(\mathbb{Z})$.

The main goal of this paper is to provide an explicit combinatorial formula for the eigenfunctions of the difference Pöschl-Teller operator $\mathcal{D}(1.1 \mathrm{a}),(1.1 \mathrm{~b})$ with integer-valued coupling parameter. For this purpose, we present a finite-difference 
version of a well-known formula due to Sato for the eigenfunctions of the reflectionless Schrödinger operators on the line [Sa, SS, DKJM, SW, OSTT, DK]. Our difference generalization of the Sato formula is derived with the aid of the inverse scattering theory of reflectionless Jacobi operators [CC, Ca2, Fla, To, Te1]. In short, the idea is to first employ inverse scattering theory to explicitly parametrize the reflectionless Jacobi operators and their corresponding Jost eigenfunctions in terms of the spectral data. By a suitable interpolation the eigenfunction formula is then extended to that of a reflectionless finite-difference operator living on the whole real line. (In general such interpolation from eigenfunctions and difference operators over $\mathbb{Z}$ to $\mathbb{R}$ is clearly not at all unique: one may for instance multiply the eigenfunctions with an arbitrary periodic function with a period equal to the step size without destroying the eigenfunction property. In our case, however, we are dealing with difference operators and eigenfunctions that (up to a similarity transformation involving an analytic function) are in essence rational in the variable. There exists therefore a natural interpolation preserving this rationality (as well as the plane-wave asymptotics of the wave functions).) Finally, to arrive at the combinatorial formula for the difference Pöschl-Teller eigenfunctions, we specialize the parameters of our difference Sato formula to the spectral data corresponding to the Jacobi operator $D(1.3 \mathrm{a}),(1.3 \mathrm{~b})$. It turns out that the expansion coefficients in the formula for the difference Pöschl-Teller eigenfunctions thus obtained may be identified as principal specializations of Schur functions. This gives rise to an interpretation of these expansion coefficients in terms of characters of irreducible representations of $S L(N ; \mathbb{Q}$, upon restriction to the principal three-dimensional subgroup $S L(2 ; \mathbb{Q}[\mathrm{Ko}]$.

Quite distinct expressions for the eigenfunctions of the difference Pöschl-Teller operator were previously found by Ruijsenaars [Ru1, Ru2]. To compare the combinatorial formula of the present paper with Ruijsenaars' formulas, we observe that it is possible to recast our eigenfunction in terms of a basic hypergeometric series. More precisely, one has to do with a $q$-version of the associated Legendre functions of integer degree. In a nutshell: our combinatorial formula yields the eigenfunction in the form of a product of a plane wave and a quotient of two $q$-polynomials (tau functions to be precise). By dividing out a common factor in the numerator and denominator one reduces the combinatorial formula to a basic hypergeometric series representation for the eigenfunction (therewith destroying the combinatorial structure in the process). That such division by a common factor is possible is not at all apparent from the explicit expressions involved: it hinges on certain nontrivial combinatorial $q$-identities (that stem from a factorization of the tau function based on the principal specialization of a $C_{N}$-type Weyl denominator formula). From the basic hypergeometric representation we arrive at Ruijsenaars' formulas via an expansion of the coefficients appearing in the basic hypergeometric series. In a sense this provides an "explanation" of the combinatorics behind the Ruijsenaars formulas within the frame work of basic hypergeometry.

A remarkable property of the eigenfunctions of $\mathcal{D}(1.1 \mathrm{a}),(1.1 \mathrm{~b})$ is the symmetry with respect to an interchange of the role of the spatial and spectral variable [Ru1, Ru2]. This symmetry, which is also referred to as self-duality, implies that the eigenfunction satisfies in essence the same difference equation in the spectral variable as it does in the spatial variable. In other words, the difference PöschlTeller problem provides an example of a difference-difference bispectral problem in the sense of Duistermaat and Grünbaum [DG, Wi, Gr, GH]. It turns out that 
the representation of the eigenfunction in terms of a basic hypergeometric series is quite helpful for inferring this bispectral symmetry.

Of special interest is the relation to the harmonic analysis on (quantum) homogeneous spaces. For $q \rightarrow 1$ the difference Pöschl-Teller eigenfunctions tend to zonal spherical functions on the hyperboloid $H^{2 N+3,1}=S O_{0}(2 N+3,1 ; \mathbb{R}) / S O_{0}(2 N+$ $2,1 ; \mathbb{R})$. This limit corresponds to the above-mentioned transition from the Ruijsenaars difference operator $\mathcal{D}(1.1 \mathrm{a}),(1.1 \mathrm{~b})$ to the Schrödinger operator with PöschlTeller potential given by $\mathcal{D}_{0}(1.2 \mathrm{~b})$. Via analytic continuation one also obtains $q$-deformations of the zonal spherical functions on the hyperboloid $H_{+}^{1,2 N+3}=$ $S O_{0}(2 N+3,1 ; \mathbb{R}) / S O(2 N+3 ; \mathbb{R})$ and the sphere $S^{2 N+3}=S O(2 N+4 ; \mathbb{R}) / S O(2 N+$ $3 ; \mathbb{R}$ ), respectively. (In the above difference and differential operators this corresponds to changing the hyperbolic cosine to hyperbolic sine or ordinary sine functions, respectively.) In the compact case (i.e. for the sphere), the $q$-deformed zonal spherical functions thus obtained boil in essence down to $q$-ultraspherical polynomials [GR]. In other words, for this case the results of the present paper amount to a new type of combinatorial formula for these polynomials. It is furthermore known that the $q$-ultraspherical polynomials themselves can again be seen as zonal spherical functions on certain quantum spheres [NM, Su]. It is therefore natural to expect that also for the noncompact cases the harmonic-analytic interpretation of the Pöschl-Teller eigenfunctions lifts to the $q$-deformed level. In particular, one expects that the eigenfunctions of the hyperbolic Ruijsenaars difference operator admit an interpretation as zonal spherical functions on certain quantum hyperboloids [Ka, KMU, SV].

The paper is structured as follows. In Section 2 the difference Sato formula is presented. Its proof by means of the inverse scattering theory of reflectionless Jacobi operators is relegated to an appendix at the end of the paper. By specialization of the spectral data, the combinatorial formula for the difference Pöschl-Teller wave functions is obtained in Section 3. Section 4 discusses basic hypergeometric representations, the bispectrality, and the relation to Ruijsenaars' formulas for the wave functions under consideration. Finally, in Section 5 our results are applied to arrive at combinatorial formulas for $q$-deformations of zonal spherical functions on certain hyperboloids and spheres. This leads, in particular, to a combinatorial representation for the $q$-ultraspherical polynomials.

Note. $i$. We shall employ the following notational conventions when referring to some standard notions from " $q$-analysis" (see e.g. [GR]).

q-Pochhammer symbols:

$$
(a ; q)_{m}= \begin{cases}1 & \text { for } m=0 \\ (1-a)(1-a q) \cdots\left(1-a q^{m-1}\right) & \text { for } m=1,2,3 \ldots\end{cases}
$$

and $\left(a_{1}, \ldots, a_{r} ; q\right)_{m}=\left(a_{1} ; q\right)_{m} \cdots\left(a_{r} ; q\right)_{m}$.

$q$-Binomial coefficients:

$$
\left(\begin{array}{l}
n \\
m
\end{array}\right)_{q}=\frac{(q ; q)_{n}}{(q ; q)_{m}(q ; q)_{n-m}}
$$

(with $n, m \in \mathbb{N}$ such that $n \geq m$ ). $q$-Numbers:

$$
[z]_{q}=\frac{q^{z / 2}-q^{-z / 2}}{q^{1 / 2}-q^{-1 / 2}}, \quad z \in \mathbb{R}(\text { or } \mathbb{Q} \text {. }
$$


q-Factorials:

$$
[m]_{q} != \begin{cases}1 & \text { for } m=0 \\ {[1]_{q}[2]_{q} \cdots[m]_{q}} & \text { for } m=1,2,3 \ldots\end{cases}
$$

Terminating basic hypergeometric series:

$$
{ }_{r+1} \phi_{r}\left[\begin{array}{r}
q^{-N}, a_{1}, \ldots, a_{r} \\
b_{1}, \ldots, b_{r}
\end{array} \mid q ; z\right]=\sum_{m=0}^{N} \frac{\left(q^{-N}, a_{1}, \ldots, a_{r}, ; q\right)_{m}}{\left(q, b_{1}, \ldots, b_{r} ; q\right)_{m}} z^{m}
$$

(with $b_{j} \neq q^{-m}$ for $m=0, \ldots, N$ and $j=1, \ldots, r$ ).

ii. Throughout the paper we shall choose the parameter $q$ such that $|q| \neq 1$.

\section{A GENERALIZED SATO FORMULA FOR FINITE-DIFFERENCE OPERATORS}

It is known that the eigenfunctions of a one-dimensional Schrödinger operator with Bargmann potential can be written explicitly with the aid of the Sato formula [Sa, SS, DKJM, SW, OSTT, DK]. In this section we present a Sato-type formula for the eigenfunctions of reflectionless finite-difference operators.

The difference operators under consideration are of the form

$$
\mathcal{D}=a(x) T+a(x-1) T^{-1},
$$

with $(T \psi)(x)=\psi(x+1)$ for $\psi: \mathbb{R} \rightarrow \mathbb{C}$ and

$$
a(x)=\sqrt{\frac{\tau(x+2) \tau(x-1)}{\tau(x+1) \tau(x)}}
$$

where

$$
\tau(x)=\sum_{J \in \subset\{1, \ldots, N\}} \prod_{j \in J} \frac{\nu_{j}}{\sinh \left(2 \kappa_{j}\right)} \prod_{\substack{j, k \in J \\ j<k}} \frac{\sinh ^{2}\left(\kappa_{j}-\kappa_{k}\right)}{\sinh ^{2}\left(\kappa_{j}+\kappa_{k}\right)} \exp \left(-2 \sum_{j \in J} \kappa_{j} x\right) .
$$

Here $0<\kappa_{1}<\cdots<\kappa_{N}$ and $\nu_{1}, \ldots, \nu_{N}>0$ are parameters encoding the spectral data of our difference operator.

Proposition 2.1 (Difference Sato formula). The wave function

$$
\psi(x, y)=\frac{\exp (x y) \chi(x, y)}{\sqrt{\tau(x+1) \tau(x-1)}}
$$

with

$$
\begin{aligned}
& \chi(x, y)= \\
& \sum_{J \in \subset\{1, \ldots, N\}} \prod_{j \in J} \frac{\nu_{j}}{\sinh \left(2 \kappa_{j}\right)} \frac{\sinh \left(y+\kappa_{j}\right)}{\sinh \left(y-\kappa_{j}\right)} \prod_{\substack{j, k \in J \\
j<k}} \frac{\sinh ^{2}\left(\kappa_{j}-\kappa_{k}\right)}{\sinh ^{2}\left(\kappa_{j}+\kappa_{k}\right)} \exp \left(-2 \sum_{j \in J} \kappa_{j} x\right),
\end{aligned}
$$

solves the difference equation

$$
a(x) \psi(x+1, y)+a(x-1) \psi(x-1, y)=\left(e^{y}+e^{-y}\right) \psi(x, y), \quad x \in \mathbb{R}
$$

(for $\left.y \neq \kappa_{j} \bmod \pi i, j=1, \ldots, N\right)$. (Here $a(x)$ and $\tau(x)$ are given by (2.2) and (2.3).) 
Proof. For $x \in \mathbb{Z}$, a proof of the difference Sato formula can be found in the appendix at the end of the paper (cf. Corollary A.2 with $n=x, z=e^{y}$ and $\left.z_{j}=e^{-\kappa_{j}}\right)$. More concretely, we have that for the stated wave function $\psi(x, y)$ our difference equation holds as an identity in the parameters $\nu_{j}$ and $\kappa_{j}$ when $x \in \mathbb{Z}$. The extension to general $x \in \mathbb{R}$ then follows from the observation that the translation $x \rightarrow x+\Delta$ is equivalent to a reparametrization of the form $\nu_{j} \rightarrow$ $\nu_{j} e^{-2 \kappa_{j} \Delta}, j=1, \ldots, N$.

Remark 2.1. The eigenfunction of Proposition 2.1 has the following reflectionless plane-wave asymptotics for $|x| \rightarrow \infty$

$$
\psi(x, y) \longrightarrow \begin{cases}\exp (x y) & \text { for } x \rightarrow+\infty \\ \exp (x y) \prod_{j=1}^{N} \frac{\sinh \left(y+\kappa_{j}\right)}{\sinh \left(y-\kappa_{j}\right)} & \text { for } x \rightarrow-\infty\end{cases}
$$

For $y=-\kappa_{j}, j=1, \ldots, N$, the wave function decays exponentially as $|x| \rightarrow \infty$. Hence, it becomes square integrable at these spectral values. The corresponding normalization constants are governed by the parameters $\nu_{j}$ :

$$
\int_{-\infty}^{\infty} \psi^{2}\left(x,-\kappa_{j}\right) \mathrm{d} x=\nu_{j}^{-1}, \quad j=1, \ldots, N .
$$

This integration formula follows from the summation formula (cf. Remark A.2 of the appendix below)

$$
\sum_{n \in \mathbb{Z}} \psi^{2}\left(n+x,-\kappa_{j}\right)=\nu_{j}^{-1}, \quad j=1, \ldots, N
$$

upon integrating in $x$ from 0 to 1 (or indeed over any interval with unit length) and interchanging the sum and integral on the l.h.s. As a matter of fact, it turns out possible to generalize the integration formula of Eq. (2.5) somewhat:

$$
\int_{-\infty}^{\infty} \psi^{2}\left(x,-\kappa_{j}\right) e^{2 \pi i m x} \mathrm{~d} x=\left\{\begin{array}{ll}
\nu_{j}^{-1} & \text { for } m=0 \\
0 & \text { for } m \in \mathbb{Z} \backslash\{0\}
\end{array},\right.
$$

$j=1, \ldots, N$. (To arrive at the latter identity, one multiplies both sides of the sum in Eq. (2.6) by $e^{2 \pi i m x}, m \in \mathbb{Z}$ before integrating in $x$.) The summation and integration formulas of Eqs. (2.6) and (2.7) are actually equivalent. Indeed, both identities are related by the Poisson summation formula.

Remark 2.2. By means of the substitution $x \rightarrow \beta^{-1} x, y \rightarrow \beta y, \kappa_{j} \rightarrow \beta \kappa_{j}$ and $\nu_{j} \rightarrow \beta \nu_{j}$, one rescales the step size of the difference equation of Proposition 2.1 to $\beta$. Division by $\beta^{2}$ and sending $\beta$ to zero then leads to the Schrödinger equation

$$
\left(\frac{\mathrm{d}^{2}}{\mathrm{~d} x^{2}}+v(x)\right) \tilde{\psi}(x, y)=y^{2} \tilde{\psi}(x, y)
$$

with a potential of the form

$$
v(x)=2 \frac{\mathrm{d}^{2}}{\mathrm{~d} x^{2}} \log (\tilde{\tau}(x))
$$

and a wave function of the form

$$
\tilde{\psi}(x, y)=\exp (x y) \frac{\tilde{\chi}(x, y)}{\tilde{\tau}(x)}
$$


where

$$
\begin{aligned}
\tilde{\chi}(x, y) & =\sum_{J \in \subset\{1, \ldots, N\}} \prod_{j \in J} \frac{\nu_{j}}{2 \kappa_{j}}\left(\frac{y+\kappa_{j}}{y-\kappa_{j}}\right) \prod_{\substack{j, k \in J \\
j<k}}\left(\frac{\kappa_{j}-\kappa_{k}}{\kappa_{j}+\kappa_{k}}\right)^{2} \exp \left(-2 \sum_{j \in J} \kappa_{j} x\right), \\
\tilde{\tau}(x) & =\sum_{J \in \subset\{1, \ldots, N\}} \prod_{j \in J} \frac{\nu_{j}}{2 \kappa_{j}} \prod_{\substack{j, k \in J \\
j<k}}\left(\frac{\kappa_{j}-\kappa_{k}}{\kappa_{j}+\kappa_{k}}\right)^{2} \exp \left(-2 \sum_{j \in J} \kappa_{j} x\right) .
\end{aligned}
$$

We thus reproduce the Sato wave function for the one-dimensional Schrödinger equation with Bargmann potential, parametrized in terms of the spectral data [Sa, SS, DKJM, SW, OSTT, DK].

Remark 2.3. Of particular interest is the situation with normalization parameters of the form

$$
\nu_{j}=\sinh \left(2 \kappa_{j}\right) \prod_{\substack{1 \leq k \leq N \\ k \neq j}}\left|\frac{\sinh \left(\kappa_{j}+\kappa_{k}\right)}{\sinh \left(\kappa_{j}-\kappa_{k}\right)}\right|, \quad j=1, \ldots, N .
$$

The difference Sato formula then simplifies to

$$
\psi(x, y)=\frac{\exp (x y) \chi(x, y)}{\sqrt{\tau(x+1) \tau(x-1)}}
$$

with

$$
\begin{aligned}
\chi(x, y) & =\sum_{J \in \subset\{1, \ldots, N\}} \prod_{j \in J} \frac{\sinh \left(y+\kappa_{j}\right)}{\sinh \left(y-\kappa_{j}\right)} \prod_{j \in J}\left|\frac{\sinh \left(\kappa_{j}+\kappa_{k}\right)}{\sinh \left(\kappa_{j}-\kappa_{k}\right)}\right| \exp \left(-2 \sum_{j \in J} \kappa_{j} x\right), \\
\tau(x) & =\sum_{J \in \subset\{1, \ldots, N\}} \prod_{j \in J}\left|\frac{\sinh \left(\kappa_{j}+\kappa_{k}\right)}{\sinh \left(\kappa_{j}-\kappa_{k}\right)}\right| \exp \left(-2 \sum_{j \in J} \kappa_{j} x\right) .
\end{aligned}
$$

For these spectral data the corresponding difference operator $\mathcal{D}(2.1)-(2.3)$ becomes parity invariant, i.e., it becomes symmetric with respect to the reflection $x \rightarrow-x, T \rightarrow T^{-1}$. Indeed, we now have that $\tau(-x)=\tau(x) e^{2 x\left(\kappa_{1}+\cdots+\kappa_{N}\right)}$, whence the coefficient $a(x)(2.2)$ enjoys the symmetry relation $a(-x-1)=a(x)$.

Remark 2.4. Via the transformation

$$
\psi(x, y)=\mathcal{A}(x) \Psi(x, y), \quad \mathcal{A}(x)=\prod_{m=1}^{\infty} a(x-m)=\sqrt{\frac{\tau(x+1)}{\tau(x-1)}},
$$

the wave function and difference equation of Proposition 2.1 pass over into

$$
\Psi(x, y)=\exp (x y) \frac{\chi(x, y)}{\tau(x+1)}
$$

and

$$
\frac{\tau(x+2) \tau(x-1)}{\tau(x+1) \tau(x)} \Psi(x+1, y)+\Psi(x-1, y)=\left(e^{y}+e^{-y}\right) \Psi(x, y) .
$$

The difference equation (2.15) with wave function (2.14) holds as a meromorphic identity in $x, y, \kappa_{j}$ and $\nu_{j}$. More specifically, one has that the equality of Eq. (2.15) is rational in $\exp (x), \exp \left(-2 \kappa_{j} x\right)$ and $\exp (y)$ as a function of $x$ and $y$, and rational in $\exp \left(\kappa_{j}\right), \exp \left(-2 \kappa_{j} x\right)$ and $\nu_{j}$ as a function of $\kappa_{j}$ and $\nu_{j}$. 


\section{Combinatorics of THE Difference Pöschl-Teller PRoblem}

The finite-difference Sato formula of the previous section is used to arrive at a combinatorial formula for the wave function of the difference Pöschl-Teller problem. The expansion coefficients turn out to be given by principal specializations of Schur characters.

We consider the following eigenvalue equation for the difference Pöschl-Teller operator $\mathcal{D}$ (1.1a),(1.1b) with $\beta=1$ and $g=N+1$ :

$$
a(x) \psi(x+1, y)+a(x-1) \psi(x-1, y)=\left(q^{y / 2}+q^{-y / 2}\right) \psi(x, y), \quad x \in \mathbb{R}
$$

with

$$
a(x)=\frac{\left(1+q^{x+N+1}\right)^{1 / 2}\left(1+q^{x-N}\right)^{1 / 2}}{\left(1+q^{x+1}\right)^{1 / 2}\left(1+q^{x}\right)^{1 / 2}}
$$

By means of the transformation

$$
\psi(x, y)=\mathcal{A}(x) \Psi(x, y), \quad \mathcal{A}(x)=\prod_{m=1}^{\infty} a(x-m)=\prod_{j=1}^{N}\left(\frac{1+q^{x+j}}{1+q^{x-j}}\right)^{1 / 2}
$$

we bring this eigenvalue equation in the rational form

$$
\begin{aligned}
\frac{\left(1+q^{x+N+1}\right)\left(1+q^{x-N}\right)}{\left(1+q^{x+1}\right)\left(1+q^{x}\right)} & \Psi(x+1, y)+\Psi(x-1, y) \\
& =\left(q^{y / 2}+q^{-y / 2}\right) \Psi(x, y), \quad x \in \mathbb{R}
\end{aligned}
$$

(cf. Remark 2.4). The following proposition provides an explicit combinatorial formula for the solution of the difference equation (3.4) with-for $|q|<1$-planewave asymptotics of the form $q^{x y / 2}$ for $x \rightarrow+\infty$. (Notice that our difference equations are invariant with respect to the inversion $q \rightarrow q^{-1}$; hence, the two cases $|q|<1$ and $|q|>1$ are equivalent, cf. Remark 3.3 below.)

Proposition 3.1 (Combinatorial representation). The wave function

$$
\Psi_{N, q}(x, y)=\frac{q^{x y / 2} \chi_{N}(x, y)}{\tau_{N}(x+1)}
$$

with

$$
\chi_{N}(x, y)=\sum_{J \subset\{1, \ldots, N\}} q^{x \sum_{j \in J} j} \prod_{j \in J} \frac{[y-j]_{q}}{[y+j]_{q}} \prod_{j \in J, k \notin J}\left|\frac{[j+k]_{q}}{[j-k]_{q}}\right|
$$

and

$$
\tau_{N}(x)=\sum_{J \subset\{1, \ldots, N\}} q^{x \sum_{j \in J} j} \prod_{j \in J, k \notin J}\left|\frac{[j+k]_{q}}{[j-k]_{q}}\right|,
$$

solves the difference equation (3.4). (Here $[z]_{q}$ represents the $q$-number $\left(q^{z / 2}\right.$ $\left.q^{-z / 2}\right) /\left(q^{1 / 2}-q^{-1 / 2}\right)$ and $y \neq-j \bmod 2 \pi i / \log q, j=1, \ldots, N$.)

Proof. The substitutions $y \rightarrow-\alpha y$ and $\kappa_{j}=\alpha j(j=1, \ldots, N)$ in the tau function and wave function of Remark 2.3 lead-upon application of the transformation in 
Remark 2.4- to the stated tau function $\tau_{N}(x)$ and wave function $\Psi_{N, q}(x, y)$ with $q=e^{-2 \alpha}$. We thus conclude that $\Psi_{N, q}(x, y)$ satisfies the difference equation

$$
\frac{\tau_{N}(x+2) \tau_{N}(x-1)}{\tau_{N}(x+1) \tau_{N}(x)} \Psi_{N, q}(x+1, y)+\Psi_{N, q}(x-1, y)=\left(q^{y / 2}+q^{-y / 2}\right) \Psi_{N, q}(x, y) .
$$

(cf. Eq. (2.15)). The proposition now follows via the factorization formula

$$
\tau_{N}(x)=\prod_{1 \leq j \leq k \leq N}\left(1+q^{x+N+1-j-k}\right)
$$

(cf. Corollary 3.4 below), from which it is readily seen that

$$
\frac{\tau_{N}(x+2) \tau_{N}(x-1)}{\tau_{N}(x+1) \tau_{N}(x)}=\frac{\left(1+q^{x+N+1}\right)\left(1+q^{x-N}\right)}{\left(1+q^{x+1}\right)\left(1+q^{x}\right)} .
$$

We will now demonstrate that the expansion coefficients $\prod_{j \in J, k \notin J} \mid[j+k]_{q} /[j-$ $k]_{q} \mid$ arise as principal specializations of certain Schur functions. Recall in this connection that for a partition $\lambda=\left(\lambda_{1}, \ldots, \lambda_{N}\right)$ (i.e. the components $\lambda_{j}$ are assumed to be ordered integers such that $\lambda_{1} \geq \lambda_{2} \geq \cdots \geq \lambda_{N} \geq 0$ ), the Schur function $s_{\lambda}\left(x_{1}, \ldots, x_{N}\right)=s_{\lambda}(\mathrm{x})$ is given explicitly by [Ma, Ch. I.3]

$$
\begin{aligned}
s_{\lambda}(\mathbf{x}) & =\frac{\operatorname{det}\left[x_{k}^{\rho_{j}+\lambda_{j}}\right]_{1 \leq j, k \leq N}}{\operatorname{det}\left[x_{k}^{\rho_{j}}\right]_{1 \leq j, k \leq N}} \\
& =\frac{\sum_{\sigma \in S_{N}}(-1)^{\sigma} x_{\sigma_{1}}^{\rho_{1}+\lambda_{1}} \cdots x_{\sigma_{N}}^{\rho_{N}+\lambda_{N}}}{\sum_{\sigma \in S_{N}}(-1)^{\sigma} x_{\sigma_{1}}^{\rho_{1}} \cdots x_{\sigma_{N}}^{\rho_{N}}}
\end{aligned}
$$

with

$$
\rho_{j}=(2 N+1-2 j) / 2, \quad j=1, \ldots, N .
$$

For our purposes the partitions of the relevant Schur functions are easiest characterized in terms of their Frobenius symbol $\left(\alpha_{1}, \ldots, \alpha_{r} \mid \beta_{1}, \ldots, \beta_{r}\right)[\mathrm{Ma}, \mathrm{Ch}$. I.1]. Here $\alpha_{j}$ and $\beta_{j}$ denote integers such that $\alpha_{1}>\alpha_{2}>\cdots>\alpha_{r} \geq 0$ and $N>\beta_{1}>\beta_{2}>\cdots>\beta_{r} \geq 0$. The corresponding partition has a Young diagram with a diagonal of $r$ boxes, such that the number of boxes in the $j^{\text {th }}$ row to the right of the diagonal box $(j, j)$ is equal to $\alpha_{j}$ and the number of boxes in the $j^{\text {th }}$ column below the diagonal box $(j, j)$ is equal to $\beta_{j}(j=1, \ldots, r)$.

Proposition 3.2 (Character formulas). Let $J=\left\{1 \leq \dot{j}_{1}<j_{2}<\cdots<j_{r} \leq N\right\}$ and let $\lambda_{J}$ be the partition with a Frobenius symbol of the form $\left(j_{r}, j_{r-1}, \ldots, j_{1} \mid j_{r}-\right.$ $\left.1, j_{r-1}-1, \ldots, j_{1}-1\right)$. Then we have that

$$
\begin{aligned}
s_{\lambda_{J}}\left(q^{\rho}\right) & =\prod_{j \in J, k \notin J}\left|\frac{[j+k]_{q}}{[j-k]_{q}}\right| \\
& =\prod_{j \in J, k \notin J}\left|\frac{1-q^{j+k}}{q^{j}-q^{k}}\right|,
\end{aligned}
$$

where $q^{\rho}=\left(q^{\rho_{1}}, \ldots, q^{\rho_{N}}\right)$ with $\rho_{1}, \ldots, \rho_{N}$ given by $(3.6)$. 
Proof. The proof is based on the hook formula (cf. [Ma, p. 44])

$$
s_{\lambda}\left(q^{\rho}\right)=\prod_{(i, j) \in \lambda} \frac{[N+c(i, j)]_{q}}{[h(i, j)]_{q}},
$$

where $c(i, j)=j-i, h(i, j)=\lambda_{i}+\lambda_{j}^{\prime}-i-j+1$, and the product is over all boxes $(i, j)$ in the Young diagram of $\lambda$. (Here $\lambda^{\prime}$ refers to the transpose of $\lambda$.) From the hook formula one deduces that

$$
\begin{aligned}
\frac{s_{\lambda_{J}}\left(q^{\rho}\right)}{s_{\lambda_{J \backslash\left\{j_{r}\right\}}}\left(q^{\rho}\right)} & =\frac{[N+c(1,1)]_{q}}{[h(1,1)]_{q}} \prod_{1<i \leq j_{r}} \frac{[N+c(i, 1)]_{q}}{[h(i, 1)]_{q}} \prod_{1<j \leq j_{r}+1} \frac{[N+c(1, j)]_{q}}{[h(1, j)]_{q}} \\
& =\frac{\left[j_{r}\right]_{q}}{\left[2 j_{r}\right]_{q}} \frac{\left[N+j_{r}\right]_{q} !}{\left[N-j_{r}\right]_{q} !} \frac{1}{\left(\left[j_{r}\right]_{q} !\right)^{2}} \prod_{1 \leq s<r} \frac{\left[j_{r}-j_{s}\right]_{q}^{2}}{\left[j_{r}+j_{s}\right]_{q}^{2}} \\
& =\prod_{k \notin J}\left|\frac{\left[j_{r}+k\right]_{q}}{\left[j_{r}-k\right]_{q}}\right|_{j \in J \backslash\left\{j_{r}\right\}}\left|\frac{\left[j+j_{r}\right]_{q}}{\left[j-j_{r}\right]_{q}}\right|^{-1}
\end{aligned}
$$

(with the notation $[m]_{q} !=[m]_{q}[m-1]_{q} \cdots[1]_{q}$ for $m$ a positive integer and $[0]_{q} !=1$ ). The proposition now follows by induction on $r$ starting from the trivial case $r=0$ (for which $s_{\lambda_{J}}\left(q^{\rho}\right)=s_{\lambda_{\vartheta}}\left(q^{\rho}\right)=s_{0}\left(q^{\rho}\right)=1$ ).

As a corollary of Proposition 3.1 and Proposition 3.2, a representation of the difference Pöschl-Teller wave function $\Psi_{N, q}(x, y)$ in terms of Schur functions is obtained.

Corollary 3.3 (Schur function representation). The wave function of the difference Pöschl-Teller problem (3.4) given by Proposition 3.1, may be written as

$$
\Psi_{N, q}(x, y)=q^{x y / 2} \frac{\sum_{\lambda} c_{\lambda}(y) s_{\lambda}\left(q^{\rho+x / 2}\right)}{\sum_{\lambda} q^{|\lambda| / 2} s_{\lambda}\left(q^{\rho+x / 2}\right)}
$$

where the sums are over all partitions $\lambda=\left(\lambda_{1}, \ldots, \lambda_{N}\right)$ with a Frobenius symbol of the form $\left(\alpha_{1}, \ldots, \alpha_{r} \mid \alpha_{1}-1, \ldots, \alpha_{r}-1\right)$ with $\alpha_{1} \leq N$,

$$
c_{\lambda}(y)=\frac{\left[y-\alpha_{1}\right]_{q}}{\left[y+\alpha_{1}\right]_{q}} \cdots \frac{\left[y-\alpha_{r}\right]_{q}}{\left[y+\alpha_{r}\right]_{q}}
$$

and $q^{\rho+x / 2}=\left(q^{\rho_{1}+x / 2}, \ldots, q^{\rho_{N}+x / 2}\right)$ with $\rho_{1}, \ldots, \rho_{N}$ given by (3.6).

Proof. One has $s_{\lambda}\left(q^{\rho+x / 2}\right)=q^{x|\lambda| / 2} s_{\lambda}\left(q^{\rho}\right)=q^{x\left(\alpha_{1}+\cdots+\alpha_{r}\right)} s_{\lambda}\left(q^{\rho}\right)$ in view of the homogeneousness of the Schur functions (here $|\lambda|=\lambda_{1}+\cdots+\lambda_{N}$ represents the number of boxes in the Young diagram of $\lambda$ ). Hence, the stated formula reduces to that of Proposition 3.1 after application of Proposition 3.2.

Another consequence of the evaluation formula for Schur functions given by Proposition 3.2, is the following factorized representation for the tau function of the difference Pöschl-Teller problem.

Corollary 3.4 (Factorization of the difference Pöschl-Teller tau function). One has that

$$
\tau_{N}(x)=\sum_{J \subset\{1, \ldots, N\}} q^{x \sum_{j \in J} j} \prod_{j \in J, k \notin J}\left|\frac{[j+k]_{q}}{[j-k]_{q}}\right|=\prod_{1 \leq j \leq k \leq N}\left(1+q^{x+\rho_{j}+\rho_{k}}\right),
$$

with $\rho_{1}, \ldots, \rho_{N}$ given by $(3.6)$. 
Proof. This factorization formula boils down to the principal specialization of the $C_{N}$-type Weyl denominator formula [Ma, p. 79]

$$
\sum_{J \subset\{1, \ldots, N\}} s_{\lambda_{J}}(\mathbf{x})=\prod_{1 \leq j \leq k \leq N}\left(1+x_{j} x_{k}\right) .
$$

Indeed, substitution of $x_{j}=q^{\rho_{j}+x / 2}, j=1, \ldots, N$ and evaluation of the Schur functions by means of Proposition 3.2 (using also the homogeneousness of the Schur functions) produces the desired factorization formula.

Remark 3.1. The identification of the expansion coefficients $\prod_{j \in J, k \notin J} \mid[j+k]_{q} /[j-$ $k]_{q} \mid$ as principal specializations of Schur functions provided by Proposition 3.2, implies that we are in fact dealing with monic symmetric unimodular Laurent polynomials in $q$ with positive integral coefficients [Ma]. Specifically, one has that

$$
\prod_{j \in J, k \notin J}\left|\frac{[j+k]_{q}}{[j-k]_{q}}\right|=\prod_{j \in J, k \notin J}\left|\frac{1-q^{j+k}}{q^{j}-q^{k}}\right|=a_{0}+a_{1}\left(q+q^{-1}\right)+\cdots+a_{d}\left(q^{d}+q^{-d}\right),
$$

where $d=\sum_{j \in J, k \notin J} \min (j, k)$ and with the numbers $a_{j}$ constituting a monotonously nonincreasing integer sequence of the form $a_{0} \geq a_{1} \geq \cdots \geq a_{d}=1$.

Remark 3.2. It is well-known that the Schur function $s_{\lambda}(\mathrm{x})$ arises as the character of the irreducible representation of the Lie group $S L(N ; \mathbb{Q}$ with highest weight determined by $\lambda$. Furthermore, the principal specialization of the Schur function $s_{\lambda}\left(q^{\rho}\right)$ corresponds from this viewpoint to the character of the restriction of the irreducible $S L(N ; \mathbb{Q}$-representation under consideration to the principal three-dimensional subgroup $S L(2 ; \mathbb{Q} \subset S L(N ; \mathbb{Q}$ [Ko]. Hence, from a representation-theoretic perspective, Proposition 3.2 states that our expansion coefficient $\prod_{j \in J, k \notin J} \mid[j+k]_{q} /[j-$ $k]_{q} \mid$ may be interpreted as the character of the irreducible representation of $S L(N ; \mathbb{Q}$ associated to the partition $\lambda_{J}$, upon restriction to the principal three-dimensional subgroup $S L(2 ; \mathbb{Q}$.

Remark 3.3. The wave function $\Psi_{N, q}(x, y)$ has the following plane-wave asymptotics for $x \rightarrow \pm \infty$ (cf. Remark 2.1)

$$
\Psi_{N, q}(x, y) \longrightarrow \begin{cases}q^{x y / 2} & \text { for } x \rightarrow+\infty \\ q^{x y / 2} \prod_{j=1}^{N}\left(\frac{1-q^{y-j}}{1-q^{y+j}}\right), & \text { for } x \rightarrow-\infty\end{cases}
$$

in case $|q|<1$ and

$$
\Psi_{N, q}(x, y) \longrightarrow \begin{cases}q^{x y / 2} \prod_{j=1}^{N}\left(\frac{1-q^{y-j}}{1-q^{y+j}}\right) & \text { for } x \rightarrow+\infty \\ q^{x y / 2} & \text { for } x \rightarrow-\infty\end{cases}
$$

in case $|q|>1$. The two cases are related by the $q \rightarrow q^{-1}$ transformation formula

$$
\Psi_{N, q^{-1}}(x, y)=\Psi_{N, q}(x,-y) \prod_{j=1}^{N} \frac{1-q^{-(y-j)}}{1-q^{-(y+j)}} .
$$

Remark 3.4. If the spatial variable $x$ in the difference equation (3.4) is being restricted to the integer lattice $\mathbb{Z}$, then one arrives at the discrete version of the Pöschl-Teller problem associated to the Jacobi operator $D(1.3 \mathrm{a}),(1.3 \mathrm{~b})$. The corresponding discrete spectral problem was studied in [SZ, Sec. 5] by means of Darboux transformations [MS]. This approach produces a shift operator that maps the 
wave functions for coupling parameter $N$ to those for coupling parameter $N+1$. In principle the eigenfunctions for any value of $N \in \mathbb{N}$ can then be obtained by acting repeatedly with the shift operator starting from the trivial case $N=0$. In practice, however, such constructive procedure soon starts to be quite cumbersome, as the expressions for the wave functions thus obtained tend to become rather complicated already after a few iterations with the shift operator. Proposition 3.1 provides a closed formula for the result of the $N$-fold iteration of the action of shift operator on a plane wave $q^{x y / 2}, x \in \mathbb{Z}$.

\section{BASIC HYPERGEOMETRY}

First the difference Pöschl-Teller eigenfunction $\Psi_{N, q}(x, y)$ given in the previous section will be recasted in terms of a basic hypergeometric series. Next we will discuss certain bispectrality properties and the relation to Ruijsenaars' wave function for the difference Pöschl-Teller model under consideration.

4.1. A basic hypergeometric representation. It turns out possible to recast the wave function of Proposition 3.1 in terms of a basic hypergeometric series. The point is that the numerator of the combinatorial formula for the wave function admits the following factorization:

$$
\begin{aligned}
\chi_{N}(x, y)= & \prod_{1 \leq j \leq k \leq N-1}\left(1+q^{x+N-j-k}\right) \\
& \times \sum_{m=0}^{N} q^{m(m+1) / 2}\left(\begin{array}{l}
N \\
m
\end{array}\right) q_{q}^{m x} \prod_{j=1}^{m}\left(\frac{1-q^{y-(N+1-j)}}{1-q^{y+j}}\right) .
\end{aligned}
$$

Comparison with the factorization of the tau function in Corollary 3.4, leads one to conclude that the numerator and denominator of $\Psi_{N, q}(x, y)$ possess a large common factor of the form $\tau_{N-1}(x)=\prod_{1 \leq j \leq k \leq N-1}\left(1+q^{x+N-j-k}\right)$. (One has that $\tau_{N}(x+1)=\tau_{N-1}(x) \prod_{j=1}^{N}\left(1+q^{x+j}\right)$.) Dividing out this common factor entails a basic hypergeometric formula. This at the price of losing sight of the underlying combinatorial structure.

Proposition 4.1 (Basic hypergeometric representation). The wave function of the difference Pöschl-Teller problem (3.4) given by Proposition 3.1 can be written as

$$
\Psi_{N, q}(x, y)=\frac{q^{x y / 2} \sum_{m=0}^{N} q^{m(m+1) / 2}\left(\begin{array}{l}
N \\
m
\end{array}\right)_{q} q^{m x} \prod_{j=1}^{m}\left(\frac{1-q^{y-(N+1-j)}}{1-q^{y+j}}\right)}{\prod_{j=1}^{N}\left(1+q^{x+j}\right)} .
$$

Proof. Substitution of a wave function of the form

$$
\Psi_{N, q}(x, y)=\frac{q^{x y / 2} \varphi(x, y)}{\prod_{j=1}^{N}\left(1+q^{x+j}\right)}
$$

in the eigenvalue equation (3.4) produces the following difference equation for the numerator function $\varphi(x, y)$

$$
q^{y}\left(1+q^{x-N}\right) \varphi(x+1, y)+\left(1+q^{x+N}\right) \varphi(x-1, y)=\left(1+q^{y}\right)\left(1+q^{x}\right) \varphi(x, y)
$$


With the Ansatz

$$
\varphi(x, y)=\sum_{m=0}^{N} c_{m} q^{m x}, \quad c_{0}=1,
$$

the difference equation for $\varphi(x, y)$ is converted into a recurrence relation for the coefficients $c_{m}$

$$
\frac{c_{m}}{c_{m-1}}=-\frac{\left(1-q^{N+1-m}\right)}{\left(1-q^{-m}\right)} \frac{\left(1-q^{y+m-N-1}\right)}{\left(1-q^{y+m}\right)}, \quad c_{0}=1 .
$$

Iteration of this recurrence entails

$$
\begin{aligned}
c_{m} & =(-1)^{m} \frac{\left(q^{N} ; q^{-1}\right)_{m}}{\left(q^{-1} ; q^{-1}\right)_{m}} \frac{\left(q^{-N+y} ; q\right)_{m}}{\left(q^{1+y} ; q\right)_{m}} \\
& =q^{m(m+1) / 2}\left(\begin{array}{l}
N \\
m
\end{array}\right)_{q} q^{m x} \prod_{j=1}^{m}\left(\frac{1-q^{y-(N+1-j)}}{1-q^{y+j}}\right) .
\end{aligned}
$$

It thus follows that the stated function solves the difference Pöschl-Teller equation (3.4). Moreover, for $|q| \neq 1$ our present function manifestly has the same asymptotics for $|x| \rightarrow \infty$ as the solution of Proposition 3.1 (cf. Remark 3.3), whence we conclude that both functions coincide as rational expressions in $q, q^{x}$ and $q^{y}$ times the plane wave $q^{x y / 2}$.

Notice that Proposition 4.1 and the factorization formula (4.1) follow from each other, given the product representation of the tau function $\tau_{N}(x)$ given by Corollary 3.4. Indeed, it is immediate from the said product representation that $\tau_{N}(x+$ 1) $=\tau_{N-1}(x) \prod_{j=1}^{N}\left(1+q^{x+j}\right)$. As indicated above, one obtains the wave function of Proposition 4.1 from that of Proposition 3.1 by plugging in the factorizations for $\chi_{N}(x, y)$ and $\tau_{N}(x+1)$ and dividing out the common factor $\tau_{N-1}(x)$ in the numerator and denominator. Reversely, equating the wave functions of Propositions 3.1 and 4.1 and multiplication of both sides by $\tau_{N}(x+1)=\tau_{N-1}(x) \prod_{j=1}^{N}\left(1+q^{x+j}\right)$ leads us to the factorization formula (4.1).

Remark 4.1. It is instructive to expand the denominator of the wave function of Proposition 4.1 by means of the $q$-binomial theorem:

$$
\Psi_{N, q}(x, y)=\frac{q^{x y / 2} \sum_{m=0}^{N} q^{m(m+1) / 2}\left(\begin{array}{l}
N \\
m
\end{array}\right)_{q} q^{m x} \prod_{j=1}^{m}\left(\frac{1-q^{y-(N+1-j)}}{1-q^{y+j}}\right)}{\sum_{m=0}^{N} q^{m(m+1) / 2}\left(\begin{array}{l}
N \\
m
\end{array}\right)_{q} q^{m x}}
$$

This way the manifest resemblance between the expressions in the numerator and denominator-which was also apparent in the combinatorial formula of Proposition 3.1-gets restored.

Remark 4.2. To see that the formula of Proposition 4.1 indeed amounts to a basic hypergeometric series as advertised, we write $\prod_{j=1}^{m}\left(1-q^{y-(N+1-j)}\right) /\left(1-q^{y+j}\right)=$ $\left(q^{-N+y} ; q\right)_{m} /\left(q^{1+y} ; q\right)_{m}$ and $q^{m(m+1) / 2}\left(\begin{array}{l}N \\ m\end{array}\right)_{q}=(-1)^{m} q^{m(N+1)}\left(q^{-N} ; q\right)_{m} /(q ; q)_{m}$ to end up with the following ${ }_{2} \phi_{1}$ representation

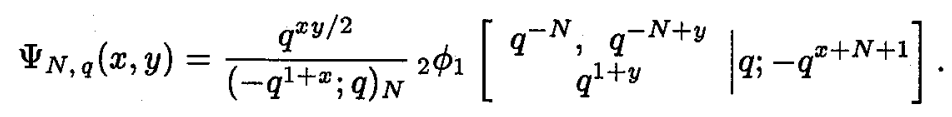


The function (4.3) constitutes a basic hypergeometric deformation of the associated Legendre function of the first kind. Substituting $x \rightarrow-2 x / \log (q)$ and $y \rightarrow-y$ in (4.3) and letting $q$ tend to 1 yields

$$
\begin{aligned}
\tilde{\Psi}_{N}(x, y) & =\frac{\exp (x y)}{\left(1+e^{-2 x}\right)^{N}} F\left(-N,-N-y ; 1-y ;-e^{-2 x}\right) \\
& =\Gamma(1-y) P_{N}^{y}(\tanh (x)),
\end{aligned}
$$

where $P_{\nu}^{\mu}(-)$ denotes the associated Legendre function of the first kind of order $\mu$ and degree $\nu$ (and $F(a, b ; c ;-)$ is the Gauss hypergeometric function). This transition corresponds to the limit from the difference Pöschl-Teller problem (3.4) to the ordinary Pöschl-Teller problem [Ti, Flu, DK]

$$
\left(\frac{\mathrm{d}^{2}}{\mathrm{~d} x^{2}}+\frac{N(N+1)}{\cosh ^{2}(x)}-y^{2}\right) \tilde{\Psi}(x, y)=0,
$$

of which $\tilde{\Psi}_{N}(x, y)(4.4)$ is a solution. In [DK] we presented a combinatorial formula for the associated Legendre function (4.4). That formula is a $q \rightarrow 1$ degeneration of the formula of Proposition 3.1.

Remark 4.3. The summation and integration formulas of Remark 2.1 give rise to corresponding identities for the difference Pöschl-Teller wave function $\Psi_{N, q}(x, y)$. Specifically, we have for $0<q<1$ that

$$
\begin{aligned}
& \sum_{n \in \mathbb{Z}} \Psi_{N, q}^{2}(n+x, j) \Delta(n+x)=\nu_{j}^{-1} \\
& \int_{-\infty}^{\infty} \Psi_{N}^{2}(x, j) \Delta(x) e^{2 \pi i m x} \mathrm{~d} x= \begin{cases}\nu_{j}^{-1} & \text { for } m=0 \\
0 & \text { for } m \in \mathbb{Z} \backslash\{0\}\end{cases}
\end{aligned}
$$

$j=1, \ldots, N$, with

$$
\Delta(x)=\prod_{j=1}^{N}\left(\frac{1+q^{x+j}}{1+q^{x-j}}\right)=\frac{\left(-q^{x+1} ; q\right)_{N}}{\left(-q^{x-1} ; q^{-1}\right)_{N}}
$$

and

$$
\begin{aligned}
\nu_{j} & =\frac{1}{2}\left|q^{j}-q^{-j}\right| \prod_{1 \leq k \leq N, k \neq j}\left|\frac{1-q^{j+k}}{q^{j}-q^{k}}\right| \\
& =\frac{\left|q^{1 / 2}-q^{-1 / 2}\right|}{2[j]_{q} ![j-1]_{q} !} \frac{[N+j]_{q} !}{[N-j]_{q} !} .
\end{aligned}
$$

(Recall in this connection that $\Psi_{N, q}(x, y)$ is obtained from the wave function of Remark 2.3 via the transformation of Remark 2.4 and setting $y \rightarrow-\alpha y, \kappa_{j}=\alpha j$ and $q=e^{-2 \alpha}$, cf. Proposition 3.1.) In the light of the previous remark, Eqs. (4.6a) and (4.6b) amount to bilinear summation and integration formulas for the basic hypergeometric function on the r.h.s. of Eq. (4.3) (cf. also Eq. (4.8) below for a ${ }_{3} \phi_{2}$ representation of this wave function).

4.2. Bispectrality. From the ${ }_{2} \phi_{1}$ representation of the wave function given by Eq. (4.3), we may pass to a ${ }_{3} \phi_{2}$ representation via a basic hypergeometric transformation formula due to Jackson [GR, Ex. 1.15 (iii) and Eq. (III.7)]

$$
{ }_{2} \phi_{1}\left[\begin{array}{c|c}
q^{-N}, b \\
c
\end{array} \mid q ; z\right]=\frac{\left(b^{-1} c ; q\right)_{N}}{(c ; q)_{N}}{ }_{3} \phi_{2}\left[\begin{array}{c}
q^{-N}, b, b c^{-1} q^{-N} z \\
b c^{-1} q^{1-N}, 0
\end{array} \mid q ; q\right] .
$$


This entails

$$
\begin{aligned}
& \Psi_{N, q}(x, y)= \\
& \quad \frac{(q ; q)_{2 N}}{(q ; q)_{N}} \frac{q^{x y / 2}}{\left(-q^{1+x}, q^{1+y} ; q\right)_{N}}{ }_{3} \phi_{2}\left[\begin{array}{c}
q^{-N}, \\
-q^{-N+x}, q^{-N+y} \\
q^{-2 N}, 0
\end{array} \mid q ; q\right] .
\end{aligned}
$$

The ${ }_{3} \phi_{2}$ representation tells us that our wave function is invariant with respect to the interchange $-q^{x} \leftrightarrow q^{y}$. But then, since the wave function solves the difference Pöschl-Teller equation (3.4), we conclude that it must also satisfy the following dual difference equation in the spectral variable:

$$
\begin{array}{r}
\frac{\left(1-q^{y+N+1}\right)\left(1-q^{y-N}\right)}{\left(1-q^{y+1}\right)\left(1-q^{y}\right)} \Psi_{N, q}(x, y+1)-\Psi_{N, q}(x, y-1) \\
=\left(q^{x / 2}-q^{-x / 2}\right) \Psi_{N, q}(x, y) .
\end{array}
$$

In other words, we are dealing with a difference-difference bispectral problem in the sense of Duistermaat and Grünbaum [DG, Wi, Gr, GH].

We can reach a perfect $x \leftrightarrow y$ symmetry in the spatial and spectral variables if we substitute $q^{x} \rightarrow-q^{x}$. This gives rise to the following modified wave function

$$
\begin{aligned}
& \hat{\Psi}_{N, q}(x, y)=\frac{(q ; q)_{2 N}}{(q ; q)_{N}} \frac{q^{x y / 2}}{\left(q^{1+x}, q^{1+y} ; q\right)_{N}} 3 \phi_{2}\left[\begin{array}{l}
q^{-N}, \\
q^{-N+x}, q^{-N+y}, 0
\end{array} \mid q ; q\right] \\
& =\frac{q^{x y / 2}}{\left(q^{1+x} ; q\right)_{N}}{ }_{2} \phi_{1}\left[\begin{array}{c}
q^{-N}, q^{-N+y}, \mid q ; q^{x+N+1} \\
q^{1+y}
\end{array}\right] \\
& =\frac{q^{x y / 2} \sum_{m=0}^{N}(-1)^{m} q^{m(m+1) / 2}\left(\begin{array}{l}
N \\
m
\end{array}\right)_{q} q^{m x} \prod_{j=1}^{m}\left(\frac{1-q^{y-(N+1-j)}}{1-q^{y+j}}\right)}{\prod_{j=1}^{N}\left(1-q^{x+j}\right)} .
\end{aligned}
$$

The modified wave function $\hat{\Psi}_{N, q}(x, y)(4.10)$ solves the difference equation

$$
\begin{array}{r}
\frac{\left(1-q^{x+N+1}\right)\left(1-q^{x-N}\right)}{\left(1-q^{x+1}\right)\left(1-q^{x}\right)} \hat{\Psi}_{N, q}(x+1, y)+\hat{\Psi}_{N, q}(x-1, y) \\
=\left(q^{y / 2}+q^{-y / 2}\right) \hat{\Psi}_{N, q}(x, y)
\end{array}
$$

and its dual counterpart with the role of $x$ and $y$ interchanged

$$
\begin{array}{r}
\frac{\left(1-q^{y+N+1}\right)\left(1-q^{y-N}\right)}{\left(1-q^{y+1}\right)\left(1-q^{y}\right)} \hat{\Psi}_{N, q}(x, y+1)+\hat{\Psi}_{N, q}(x, y-1) \\
=\left(q^{x / 2}+q^{-x / 2}\right) \hat{\Psi}_{N, q}(x, y) .
\end{array}
$$

Remark 4.4. We know from Remark 4.2 that after substituting $x \rightarrow-2 x / \log (q)$ and $y \rightarrow-y$ in the wave function $\Psi_{N, q}(x, y)$, we recover the associated Legendre function $\tilde{\Psi}_{N}(x, y)(4.4)$ in the limit $q \rightarrow 1$. The difference equation in the spectral variable given by Eq. (4.9) then degenerates to

$$
\begin{array}{r}
\tilde{\Psi}_{N}(x, y+1)-\frac{(N+y)(N+1-y)}{y(1-y)} \tilde{\Psi}_{N}(x, y-1) \\
=\left(e^{x}-e^{-x}\right) \tilde{\Psi}_{N}(x, y)
\end{array}
$$


The same $q \rightarrow 1$ transition applied to the modified wave function $\hat{\Psi}_{N, q}(x, y)(4.10)$ produces

$$
\tilde{\hat{\Psi}}_{N}(x, y)=\frac{\exp (x y)}{\left(1-e^{-2 x}\right)^{N}} F\left(-N,-N-y ; 1-y ; e^{-2 x}\right) .
$$

The corresponding degeneration of the bispectral pair (4.11a), (4.11b) becomes

$$
\begin{array}{r}
\left(\frac{\mathrm{d}^{2}}{\mathrm{~d} x^{2}}-\frac{N(N+1)}{\sinh ^{2}(x)}-y^{2}\right) \tilde{\hat{\Psi}}_{N}(x, y)=0, \\
y(1-y) \\
=\left(e^{x}+e^{-x}\right) \tilde{\hat{\Psi}}_{N}(x, y) .
\end{array}
$$

Remark 4.5. The combinatorial and Schur function representations for the modified wave function $\hat{\Psi}_{N, q}(x, y)(4.10)$ read

$$
\hat{\Psi}_{N, q}(x, y)=q^{x y / 2} \frac{\sum_{J \subset\{1, \ldots, N\}}(-1)^{\sum_{j \in J} j} q^{x \sum_{j \in J}^{j}} \prod_{j \in J} \frac{[y-j]_{q}}{[y+j]_{q}} \prod_{j \in J, k \notin J}\left|\frac{[j+k]_{q}}{[j-k]_{q}}\right|}{\sum_{J \subset\{1, \ldots, N\}}(-1)^{\sum_{j \in J} j} q^{(x+1) \sum_{j \in J}^{j}} \prod_{j \in J, k \notin J}\left|\frac{[j+k]_{q}}{[j-k]_{q}}\right|}
$$

and

$$
\hat{\Psi}_{N, q}(x, y)=q^{x y / 2} \frac{\sum_{\lambda}(-1)^{|\lambda| / 2} c_{\lambda}(y) s_{\lambda}\left(q^{\rho+x / 2}\right)}{\sum_{\lambda}(-q)^{|\lambda| / 2} s_{\lambda}\left(q^{\rho+x / 2}\right)}
$$

where the notation is in correspondence with Proposition 3.1 and Corollary 3.3, respectively.

Remark 4.6. It is not so difficult to deduce the bispectral symmetry of the wave functions $\Psi_{N, q}(x, y)$ and $\hat{\Psi}_{N, q}(x, y)$ in the spatial and spectral variables more directly, without having to rely on the Jackson transformation formula (4.7). To see this, let us substitute the Ansatz

$$
\hat{\Psi}_{N, q}(x, y)=\frac{q^{x y / 2} \sum_{m=0}^{N} C_{m}(y) q^{m x}}{\prod_{j=1}^{N}\left(1-q^{x+j}\right)}
$$

in the dual difference equation (4.11b). We obtain the following difference-difference relation involving the coefficients $C_{m}(q)$ :

$$
\frac{\left(1-q^{y+N+1}\right)\left(1-q^{y-N}\right)}{\left(1-q^{y}\right)\left(1-q^{y+1}\right)} C_{m-1}(y+1)+C_{m}(y-1)=C_{m-1}(y)+C_{m}(y)
$$

for $m=0, \ldots, N+1$ with the boundary conventions $C_{-1}(y)=C_{N+1}(y)=0$. One readily checks that

$$
\begin{aligned}
C_{m}(y) & =\frac{\left(q^{N} ; q^{-1}\right)_{m}}{\left(q^{-1} ; q^{-1}\right)_{m}} \frac{\left(q^{-N+y} ; q\right)_{m}}{\left(q^{1+y} ; q\right)_{m}} \\
& =(-1)^{m} q^{m(m+1) / 2}\left(\begin{array}{l}
N \\
m
\end{array}\right)_{q} \prod_{j=1}^{m}\left(\frac{1-q^{y-(N+1-j)}}{1-q^{y+j}}\right)
\end{aligned}
$$

$m=0, \ldots, N$, constitutes a solution of relation (4.18). (We have normalized our solution such that $C_{0}(y)=1$.) This verifies in a direct manner (without invoking 
of hypergeometric transformations or a priori use of the bispectral symmetry) that the modified wave function $\hat{\Psi}_{N, q}(x, y)$ on the second/third line of (4.10) satisfies the dual difference equation (4.11b). In other words, we are dealing with a wave function $\hat{\Psi}_{N, q}(x, y)$ which: (i) has the structure of a plane wave $q^{x y / 2}$ times a rational expression in $q^{x}, q^{y}$ and $q$, (ii) satisfies two difference equations (4.11a) and $(4.11 \mathrm{~b})$ in the spatial and spectral variable, respectively, that are related by an interchange of the role of $x$ and $y$, (iii) has plane-wave asymptotics of the form $q^{x y / 2}$ for either $q^{x}$ or $q^{y}$ going to zero. But then $\hat{\Psi}_{N, q}(x, y)$ must actually be symmetric in $x$ and $y$ (roughly speaking because it satisfies the "same" difference equations plus asymptotic boundary conditions in $x$ and $y)$ and, equivalently, $\Psi_{N, q}(x, y)$ must be invariant with respect to the interchange $-q^{x} \leftrightarrow q^{y}$.

4.3. Relation to Ruijsenaars' wave functions. Quite distinct looking representations for the eigenfunctions of the difference Pöschl-Teller system were found previously by Ruijsenaars [Ru1, Ru2]. The following proposition establishes a relation between our modified wave function $\hat{\Psi}_{N, q}(x, y)(4.10)$ and the formulas presented by Ruijsenaars.

Proposition 4.2 (Ruijsenaars-type representation). The modified wave function $\hat{\Psi}_{N, q}(x, y)(4.10)$ can be written as

$$
\hat{\Psi}_{N, q}(x, y)=\frac{q^{x y / 2} \sum_{0 \leq m, n \leq N}(-1)^{m+n} q^{m x+n y} c_{m}(q) s_{m, n}(q)}{\prod_{1 \leq j \leq N}\left(1-q^{x+j}\right)\left(1-q^{y+j}\right)},
$$

with

$$
c_{m}(q)=q^{m(m+1) / 2}\left(\begin{array}{l}
N \\
m
\end{array}\right)_{q}=\sum_{1 \leq i_{1}<\cdots<i_{m} \leq N} q^{\left(i_{1}+\cdots+i_{m}\right)}
$$

and

$$
s_{m, n}(q)=\sum_{\substack{i_{1}, \ldots, i_{m} \in\{-N, \ldots,-N-1+m, m+1, \ldots, N\} \\ i_{1}<\cdots<i_{n}}} q^{\left(i_{1}+\cdots+i_{n}\right)} .
$$

Proof. It is clear that

$$
\prod_{j=1}^{m}\left(1-q^{y-(N+1-j)}\right) \prod_{j=m+1}^{N}\left(1-q^{y+j}\right)=\sum_{n=0}^{N}(-1)^{n} s_{m, n}(q) q^{n y}
$$

with $s_{m, n}(q)$ as stated in the proposition. Indeed, the expansion coefficient $s_{m, n}(q)$ on the r.h.s. of (4.20) boils down to the sum of the $n^{\text {th }}$ order principal minors of the $N$-dimensional diagonal matrix $\operatorname{diag}\left(q^{-N}, \ldots, q^{-N-1+m}, q^{m+1}, \ldots, q^{N}\right)$. From a comparison between the $q$-binomial theorem

$$
\prod_{j=1}^{N}\left(1-q^{y+j}\right)=\sum_{n=0}^{N}(-1)^{n} q^{m(m+1) / 2}\left(\begin{array}{c}
N \\
n
\end{array}\right)_{q} q^{n y}
$$

and the expansion in (4.20) for $m=0$, we learn that

$$
q^{m(m+1) / 2}\left(\begin{array}{l}
N \\
m
\end{array}\right)_{q}=s_{0, m}(q)=\sum_{1 \leq i_{1}<\cdots<i_{m} \leq N} q^{\left(i_{1}+\cdots+i_{m}\right)} .
$$


The proposition now readily follows upon multiplication of both the numerator and denominator of $\hat{\Psi}_{N, q}(x, y)(4.10)$ by a factor $\left(1-q^{y+1}\right) \cdots\left(1-q^{y+N}\right)$ and expansion of all products and $q$-binomials arising in the resulting numerator with the aid of Eqs. (4.20) and (4.21).

The formula for the wave function $\hat{\Psi}_{N, q}(x, y)$ in Proposition 4.2 compares with Ruijsenaars' expressions for the eigenfunctions of the difference Pöschl-Teller problem. From these formulas Ruijsenaars first established the wave functions' bispectral $x \leftrightarrow y$ symmetry by investigating corresponding symmetry properties of the expansion coefficients $c_{m}(q)$ and $s_{m, n}(q)$ [Ru1, Ru2].

\section{5. $q$-SPHERICAL FUNCTIONS}

In this section we use our expressions for the difference Pöschl-Teller wave functions to arrive at combinatorial formulas for basic hypergeometric deformations of zonal spherical functions on certain odd-dimensional hyperboloids and spheres.

5.1. Combinatorial formulas. Multiplication of $\hat{\Psi}_{N, q}(x, y)(4.10)$ by the factor $q^{(x+y)(N+1) / 2} / \prod_{j=0}^{N}\left(1-q^{x-j}\right)\left(1-q^{y-j}\right)$ entails a wave function of the form

$$
\frac{\sum_{m=0}^{N}(-1)^{m} q^{m(m+1) / 2}\left(\begin{array}{l}
N \\
m
\end{array}\right)_{q} q^{m x} \prod_{j=1}^{m}\left(1-q^{y-(N+1-j)}\right) \prod_{j=m+1}^{N}\left(1-q^{y+j}\right)}{\prod_{j=-N}^{N}\left(1-q^{x+j}\right)\left(1-q^{y+j}\right)} .
$$

The difference equations (4.11a) and (4.11b) induce the following bispectral pair for this wave function:

$$
\begin{array}{r}
t^{-1 / 2}\left(\frac{1-q^{x} t}{1-q^{x}}\right) \Phi_{N, q}(x+1, y)+t^{1 / 2}\left(\frac{1-q^{x} t^{-1}}{1-q^{x}}\right) \Phi_{N, q}(x-1, y) \\
=\left(q^{y / 2}+q^{-y / 2}\right) \Phi_{N, q}(x, y) \\
t^{-1 / 2}\left(\frac{1-q^{y} t}{1-q^{y}}\right) \Phi_{N, q}(x, y+1)+t^{1 / 2}\left(\frac{1-q^{y} t^{-1}}{1-q^{y}}\right) \Phi_{N, q}(x, y-1) \\
=\left(q^{x / 2}+q^{-x / 2}\right) \Phi_{N, q}(x, y)
\end{array}
$$

where $t=q^{N+1}$.

From Remark 4.5 we can extract a combinatorial representation and a formula in terms of Schur functions for $\Phi_{N, q}(x, y)(5.1)$. The combinatorial representation at issue reads

$$
\begin{gathered}
\Phi_{N, q}(x, y)=q^{x y / 2+(N+1)(x+y) / 2} \prod_{j=-N}^{N}\left(1-q^{x+j}\right)^{-1}\left(1-q^{y+j}\right)^{-1} \times \\
\frac{\sum_{J \subset\{1, \ldots, N\}}(-1)^{\sum_{j \in J} j} q^{(x+1) \sum_{j \in J} j} \prod_{j \in J, k \notin J}\left|\frac{[j+k]_{q}}{[j-k]_{q}}\right| \prod_{j \in J}\left(1-q^{y-j}\right) \prod_{k \notin J}\left(1-q^{y+k}\right)}{\sum_{J \subset\{1, \ldots, N-1\}}(-1)^{\sum_{j \in J}^{j}} q^{x \sum_{j \in J}^{j}} \prod_{j \in J, k \notin J}\left|\frac{[j+k]_{q}}{[j-k]_{q}}\right|} .
\end{gathered}
$$

If preferred, one may also absorb (parts of) the prefactor on the first line of Eq. (5.3) into the main part of the formula given by the second line. For instance, 
absorbtion of a factor of the form $\prod_{j=1}^{N}\left(1-q^{x+j}\right)^{-1}$ is taken into account by means of the substitutions $N \rightarrow N+1$ and $x \rightarrow x+1$ in the denominator of the second line (so, in particular, the summation in the denominator becomes over all subsets $J \subset\{1, \ldots, N\})$. Similarly, absorbtion of the whole factor $\prod_{j=-N}^{N}\left(1-q^{x+j}\right)^{-1}$ into the second line gives rise to the substitution $N \rightarrow N+2$ in the denominator (so the summation in the denominator becomes over all subsets $J \subset\{1, \ldots, N+1\}$ ). Absorbtion properties of this type are readily inferred by means of the product formula for the denominator stemming from Corollary 3.4. Specifically, one readsoff from Corollary 3.4 that

$$
\frac{\tau_{N}(x+1)}{\tau_{N-1}(x)}=\prod_{j=1}^{N}\left(1+q^{x+j}\right) \quad \text { and } \quad \frac{\tau_{N+1}(x)}{\tau_{N-1}(x)}=\prod_{j=-N}^{N}\left(1+q^{x+j}\right) .
$$

These identities give rise to the above-metioned absorbtion properties upon changing $q^{x}$ to $-q^{x}$. Factors of the form $\prod_{j=1}^{N}\left(1-q^{y+j}\right)^{-1}$ and $\prod_{j=-N}^{N}\left(1-q^{y+j}\right)^{-1}$ are moreover conveniently absorbed into the product $\prod_{j \in J}\left(1-q^{y-j}\right) \prod_{k \notin J}\left(1-q^{y+k}\right)$, appearing in the numerator of the second line. An advantage of the stated representation given by Eq. (5.3) is, however, that it is well-suited to reflect the symmetry in $x$ and $y$. Indeed, the expression on the second line of Eq. (5.3) represents a symmetric polynomial in $q^{x}$ and $q^{y}$, which coincides with the numerator on the second line of Eq. (5.1). (This equality - which at this point simply amounts to the observation that Eqs. (5.1) and (5.3) represent the same wave function-goes in fact back to the factorization formula of Eq. (4.1) upon division by $\prod_{j=0}^{N}\left(1-q^{y-j}\right)$ and substitution of $q^{x} \rightarrow-q^{x}$.)

When casted in terms of Schur functions we obtain the formula (cf. Remark 4.5)

$$
\begin{gathered}
\Phi_{N, q}(x, y)=q^{x y / 2+(N+1)(x+y) / 2} \prod_{j=-N}^{N}\left(1-q^{x+j}\right)^{-1}\left(1-q^{y+j}\right)^{-1} \\
\times \frac{\sum_{\lambda}(-q)^{|\lambda| / 2} b_{\lambda}(y) s_{\lambda}\left(q^{\rho(N)+x / 2}\right)}{\sum_{\mu}(-1)^{|\mu| / 2} s_{\mu}\left(q^{\rho(N-1)+x / 2}\right)}
\end{gathered}
$$

Here the sums in the numerator and denominator are over all partitions $\lambda=$ $\left(\lambda_{1}, \ldots, \lambda_{N}\right)$ and $\mu=\left(\mu_{1}, \ldots, \mu_{N-1}\right)$ characterized by a Frobenius symbol of the form $\left(\alpha_{1}, \ldots, \alpha_{r} \mid \alpha_{1}-1, \ldots, \alpha_{r}-1\right)$, with for the numerator $\alpha_{1} \leq N$ (and $r=0, \ldots, N)$ and for the denominator $\alpha_{1} \leq N-1$ (and $r=0, \ldots, N-1$ ). The coefficient $b_{\lambda}(y)$ is given by

$$
b_{\lambda}(y)=\left(1-q^{y-\alpha_{1}}\right) \cdots\left(1-q^{y-\alpha_{r}}\right)\left(1-q^{y+\beta_{1}}\right) \cdots\left(1-q^{\beta_{N-r}}\right),
$$

where the integers $\beta_{1}, \ldots, \beta_{N-r}$ complement the integers $\alpha_{1}, \ldots, \alpha_{r}$ such that $\left\{\alpha_{1}, \ldots, \alpha_{r}, \beta_{1}, \ldots, \beta_{N-r}\right\}=\{1, \ldots, N\}$. (We have also used the notation $\rho(N)=$ $(N-1, N-3, \ldots, N+1-2 j, \ldots,-N+3,-N+1)$ and $\rho(N-1)=(N-2, N-$ $4, \ldots, N-2 j, \ldots,-N+4,-N+2)$ to distinguish the $N$ - and $(N-1)$-dimensional rho vectors used in numerator and denominator, respectively.)

5.2. Regularity. The wave function $\Phi_{N, q}(x, y)$ becomes singular for $x$ (or $y$ ) equal to $-N,-N+1, \ldots, N-1, N \bmod 2 \pi i / \log (q)$. This means in particular that we have singularities on the real line. Restriction to the even component produces a solution of the bispectral pair (5.2a), (5.2b) that is regular for $x, y$ on the real line. 
Proposition 5.1 (Regular wave function). The even combination of wave functions $\Phi_{N, q}(x, y)+\Phi_{N, q}(-x, y)$ constitutes a solution of the bispectral pair (5.2a), (5.2b) that is regular for $x, y \in \mathbb{R}$ and symmetric with respect to the interchange $x \leftrightarrow y$.

Proof. One has that $\Phi_{N, q}(-x,-y)=\Phi_{N, q}(x, y)$. To see this let us substitute $x \rightarrow-x$ and $y \rightarrow-y$ into the representation of Eq. (5.1). Multiplication of the resulting numerator and denominator by $q^{(x+y)(2 N+1)}\left(=q^{(x+y)(N+1)} q^{(x+y) N}\right)$ and changing of the summation variable in the numerator from $m$ to $N-m$, readily reproduces the expression on the r.h.s. of Eq. (5.1). (One pulls a factor $q^{(x+y) N}$ into the sum in the numerator and a factor $q^{(x+y)(2 N+1)}$ into the products of the denominator.) From this $(x, y) \rightarrow(-x,-y)$ symmetry it follows that the even combination $\Phi_{N, q}(x, y)+\Phi_{N, q}(-x, y)$ inherits the bispectral $x \leftrightarrow y$ invariance from $\Phi_{N, q}(x, y)$. It is furthermore clear that $\Phi_{N, q}(x, y)+\Phi_{N, q}(-x, y)$ solves Eq. (5.2b) since $\Phi_{N, q}(x, y)$ does so. (Notice in this connection that the eigenvalue on the r.h.s. of Eq. (5.2b) is even in $x$.) But then the even combination must in fact also satisfy Eq. (5.2a) in view of the mentioned $x \leftrightarrow y$ symmetry of the wave function.

It remains to show that the solution $\Phi_{N, q}(x, y)+\Phi_{N, q}(-x, y)$ is regular for $x, y$ on the real line. In view of the evenness and the $x \leftrightarrow y$ symmetry it suffices to check that the numerator of

$$
\begin{aligned}
& \Phi_{N, q}(x, y)+\Phi_{N, q}(-x, y)=q^{(2 N+1) x / 2+(N+1) y / 2} \times \\
& \frac{\sum_{m=0}^{N}(-1)^{m} q^{m(m+1) / 2}\left(\begin{array}{l}
N \\
m
\end{array}\right)_{q}\left(q^{x(y+2 m-N) / 2}-q^{-x(y+2 m-N) / 2}\right) b_{m}(y)}{\prod_{j=-N}^{N}\left(1-q^{x+j}\right)\left(1-q^{y+j}\right)}
\end{aligned}
$$

where

$$
b_{m}(y)=\prod_{j=1}^{m}\left(1-q^{y-(N+1-j)}\right) \prod_{j=m+1}^{N}\left(1-q^{y+j}\right),
$$

vanishes at $y=0,1, \ldots, N$. By means of some straightforward manipulations it is seen that for $y=k$, with $k \in\{0, \ldots, N\}$, the numerator on the second line of Eq. (5.6) reduces to

$$
\sum_{m=0}^{N-k} d_{m}\left(q^{-x(N-k-2 m) / 2}-q^{x(N-k-2 m) / 2}\right)
$$

with

$$
d_{m}=\frac{q^{-m(N-k-m)}(q ; q)_{N}(q ; q)_{N+k}(q ; q)_{N-k}}{(q ; q)_{m}(q ; q)_{N-k-m}(q ; q)_{N-m}(q ; q)_{k+m}} .
$$

The terms of the sum in Eq. (5.7) cancel pairwise due to the $d_{N-k-m}=d_{m}$ symmetry property of the coefficients. We thus have that the poles at $x, y \in\{-N,-N+$ $1, \ldots, N-1, N\}$, caused by the denominators of $\Phi_{N, q}(x, y)$ and $\Phi_{N, q}(-x, y)$, cancel each other in the even combination $\Phi_{N, q}(x, y)+\Phi_{N, q}(-x, y)$ due to zeros arising from the numerator. 
5.3. $q$-Ultraspherical polynomials. It is evident from Eq. (5.1) that for $y=$ $k \in \mathbb{Z} \backslash\{-N,-N+1, \ldots, N-1, N\}$ the wave function $\Phi_{N, q}(x, y)$ becomes rational in $q^{x / 2}$ and (anti-)periodic with respect to the (complex) translation $x \rightarrow$ $x+2 \pi i / \log (q)$. (One has that $\Phi_{N, q}(x+2 \pi i / \log (q), k)=(-1)^{N+1+k} \Phi_{N, q}(x, k)$.) We therefore conclude that the even combination $\Phi_{N, q}(x, y)+\Phi_{N, q}(-x, y)$ becomes an entire function in $x$ at such integer values of the spectral parameter $y$. Indeed, now the residues of all poles congruent to $x=-N,-N+1, \ldots, N-1, N$ $\bmod 2 \pi i / \log (q)$-originating from the denominators of $\Phi_{N, q}( \pm x, k)$-vanish in the even combination because of Proposition 5.1 (which guarantees the vanishing of the residues at the real poles) and the said (anti-)periodicity in $x$. As an entire rational function in $q^{x / 2}$ it is clear that $\Phi_{N, q}(x, k)+\Phi_{N, q}(-x, k)$ must in fact be a Laurent polynomial in $q^{x / 2}$. The evenness moreover implies that we are dealing with a polynomial in $q^{x / 2}+q^{-x / 2}$. The polynomial in question turns out to be a (renormalized) $q$-ultraspherical polynomial. Let us recall at this point that the $q$-ultraspherical polynomial $C_{n}^{g}(-; q)$ (with parameter $g$ and degree $n \in \mathbb{N}$ ) may be written explicitly as [GR, Ch. 7.4]

$$
C_{n}^{g}(\cosh (\alpha x) ; q)=\sum_{m=0}^{n} \frac{\left(q^{g} ; q\right)_{m}}{(q ; q)_{m}} \frac{\left(q^{g} ; q\right)_{n-m}}{(q ; q)_{n-m}} q^{(m-n / 2) x}, \quad q=e^{-2 \alpha}
$$

Alternatively, one may also represent the $q$-ultraspherical polynomial in terms of a terminating ${ }_{3} \phi_{2}$ series [GR, Ex. 1.29]

$$
C_{n}^{g}(\cosh (\alpha x) ; q)=q^{-n g / 2} \frac{\left(q^{2 g} ; q\right)_{n}}{(q ; q)_{n}} P_{n}^{g}(\cosh (\alpha x) ; q), \quad q=e^{-2 \alpha}
$$

with

$$
P_{n}^{g}(\cosh (\alpha x) ; q)=q^{n(x-g) / 2}{ }_{3} \phi_{2}\left[\begin{array}{c}
q^{-n}, q^{g}, q^{g-x} \\
q^{2 g}, 0^{2}
\end{array} \mid q ; q\right] .
$$

Here $P_{n}^{g}(\cosh (\alpha x) ; q)$ denotes the renormalized $q$-ultraspherical polynomial with a normalization such that $P_{n}^{g}(\cosh (\alpha g) ; q)=1$.

Proposition 5.2 ( $q$-Ultraspherical polynomials). One has that

$$
\begin{gathered}
c_{N} P_{n}^{N+1}(\cosh (\alpha x) ; q)= \\
\Phi_{N, q}(x, n+N+1)+\Phi_{N, q}(-x, n+N+1), \quad n \in \mathbb{N}, \\
\text { with } c_{N}=(-1)^{N+1} q^{(N+1)(2 N+1) / 2}(q ; q)_{N} /(q ; q)_{2 N+1}\left(\text { and } q=e^{-2 \alpha}\right) .
\end{gathered}
$$

Proof. We know (cf. above) that $p_{n}(x) \equiv \Phi_{N, q}(x, n+N+1)+\Phi_{N, q}(-x, n+N+$ 1 ) is a polynomial in $q^{x / 2}+q^{-x / 2}$. The degree of this polynomial follows from the asymptotics at infinity. Specifically, from Eq. (5.1) we read-off the following asymptotics for $q^{x} \rightarrow 0^{\prime}$ (so $q^{-x} \rightarrow \infty$ )

$$
\Phi_{N, q}( \pm x, y) \longrightarrow q^{ \pm x y / 2+(N+1)(x \pm y) / 2} \prod_{j=0}^{N}\left(1-q^{ \pm y-j}\right)^{-1}
$$

and thus

$$
\begin{aligned}
p_{n}(x) & \longrightarrow q^{-n x / 2-(N+1)(n+N+1) / 2} \prod_{j=0}^{N}\left(1-q^{-n-N-1-j}\right)^{-1} \\
& =(-1)^{N+1} q^{-n x / 2} q^{(N+1)(n+2 N+1) / 2} /\left(q^{n+N+1} ; q\right)_{N+1}
\end{aligned}
$$


Hence, $p_{n}(x)$ is a polynomial of degree $n$ in $q^{x / 2}+q^{-x / 2}$. Specialization of the spectral parameter to $y=n+N+1$ in Eq. (5.2a) gives rise the following difference equation for $p_{n}(x)$

$$
\begin{array}{r}
q^{-(N+1) / 2}\left(\frac{1-q^{x+N+1}}{1-q^{x}}\right) p_{n}(x+1)+q^{(N+1) / 2}\left(\frac{1-q^{x-N-1}}{1-q^{x}}\right) p_{n}(x-1) \\
=2 \cosh (\alpha(n+N+1)) p_{n}(x) .
\end{array}
$$

It is not difficult to infer that the $q$-ultraspherical polynomial $C_{n}^{N+1}(\cosh (\alpha x) ; q)$ also satisfies this difference equation. One quick way to see this is by substituting the representation of Eq. (5.8). (In other words, Eq. (5.11) boils down to the second-order difference equation for the $q$-ultraspherical polynomials.) But then $p_{n}(x)$ must be proportional to $C_{n}^{N+1}(\cosh (\alpha x) ; q)$ (and hence to $P_{n}^{N+1}(\cosh (\alpha x) ; q)$ (5.9a)), since both polynomials are eigenfunctions of the second-order difference operator determined by the first line of Eq. (5.11) corresponding to the same eigenvalue. (Here one also uses that the eigenvalues $2 \cosh (\alpha(n+N+1)), n \in \mathbb{N}$ are nondegenerate if $\operatorname{Re}(\alpha) \neq 0$, i.e. $|q| \neq 1$.) The proportionality constant follows from the asymptotics for $q^{x} \rightarrow 0$. Indeed, comparing the asymptotics (cf. Eq. $(5.8))$

$$
C_{n}^{N+1}(\cosh (\alpha x) ; q) \longrightarrow q^{-n x / 2}\left(q^{N+1} ; q\right)_{n} /(q ; q)_{n}, \quad \text { for } q^{x} \rightarrow 0,
$$

with that of Eq. (5.10) entails (using also Eq. (5.9a)) that $p_{n}(x)=c_{N} P_{n}^{N+1}(\cosh (\alpha x)$ ) with

$$
\begin{aligned}
c_{N} & =(-1)^{N+1} q^{(N+1)(2 N+1) / 2}\left(q^{2 N+2} ; q\right)_{n}\left(q^{N+1} ; q\right)_{n}^{-1}\left(q^{n+N+1} ; q\right)_{N+1}^{-1} \\
& =(-1)^{N+1} q^{(N+1)(2 N+1) / 2}(q ; q)_{N} /(q ; q)_{2 N+1} .
\end{aligned}
$$

Proposition 5.2 provides us with three alternative formulas for the $q$-ultraspherical polynomials of the form $c_{N} P_{n}^{N+1}(\cosh (\alpha x) ; q)=\Phi_{N, q}(x, n+N+1)+\Phi_{N, q}(-x, n+$ $N+1$ ). Specifically, by taking $\Phi_{N, q}(x, y)$ (i) from Eq. (5.1), (ii) from Eq. (5.3), or (iii) from Eqs. (5.5a), (5.5b), we arrive at (i) a representation in terms of (in essence) $q$-analogs of the integer-degree associated Legendre functions (cf. Remark 4.2), (ii) a combinatorial representation, or (iii) a representation in terms of Schur functions, respectively. These formulas are $q$-deformations of corresponding formulas for the ultraspherical polynomials $(q=1)$ that were presented in [DK]. By expansion of the products and $q$-binomials in the numerator of Eq. (5.1) (cf. the proof of Proposition 4.2), we can rewrite the first of our three formulas as an expression of the type $c_{N} P_{n}^{N+1}(\cosh (\alpha x) ; q)=\Phi_{N, q}(x, n+N+1)+\Phi_{N, q}(-x, n+N+1)$ with

$$
\Phi_{N, q}(x, y)=\frac{q^{x y / 2+(N+1)(x+y) / 2} \sum_{0 \leq m, n \leq N}(-1)^{m+n} q^{m x+n y} s_{0, m}(q) s_{m, n}(q)}{\prod_{-N \leq j \leq N}\left(1-q^{x+j}\right)\left(1-q^{y+j}\right)},
$$

where

$$
s_{m, n}(q)=\sum_{\substack{i_{1}, \ldots, i_{m} \in\{-N, \ldots,-N-1+m, m+1, \ldots, N\} \\ i_{1}<\cdots<i_{n}}} q^{\left(i_{1}+\cdots+i_{n}\right)} .
$$

In essence this last representation boils down to an expression for the $q$-ultraspherical polynomials previously obtained by Ruijsenaars [Ru2]. 
Remark 5.1. It was used in the proof of Proposition 5.2 that for $y=N+1+n$ the first equation of the bispectral pair $(5.2 \mathrm{a}),(5.2 \mathrm{~b})$ goes over in the second-order difference equation for the $q$-ultraspherical polynomials (cf. Eq. (5.11)). From the second equation of the bispectral pair one obtains with this substitution a recurrence relation for the polynomials $p_{n}(x)=\Phi_{N, q}(x, n+N+1)+\Phi_{N, q}(-x, n+N+1)$ :

$$
\begin{gathered}
q^{-(N+1) / 2}\left(\frac{1-q^{n+2 N+2}}{1-q^{n+N+1}}\right) p_{n+1}(x)+q^{(N+1) / 2}\left(\frac{1-q^{n}}{1-q^{n+N+1}}\right) p_{n-1}(x) \\
=2 \cosh (\alpha x) p_{n}(x)
\end{gathered}
$$

By passing to the renormalized polynomials

$$
c_{n}(x)=p_{n}(x) q^{-n(N+1) / 2}\left(q^{2 N+2} ; q\right)_{n} /(q ; q)_{n}
$$

this recurrence relation is brought to the form

$$
\left(\frac{1-q^{n+1}}{1-q^{n+N+1}}\right) c_{n+1}(x)+\left(\frac{1-q^{2 N+1+n}}{1-q^{n+N+1}}\right) c_{n-1}(x)=2 \cosh (\alpha x) c_{n}(x) .
$$

The latter relation boils down to the well-known three-term recurrence relation for the $q$-ultraspherical polynomials $C_{n}^{N+1}(\cosh (\alpha x) ; q)$ [GR, Ex. 7.19]. (Notice in this connection that $c_{n}(x)=c_{N} C_{n}^{N+1}(\cosh (\alpha x) ; q)$ with the proportionality constant $c_{N}$ being taken from Proposition 5.2.)

5.4. Zonal spherical functions. It is known that $q$-ultraspherical polynomials appear in the harmonic analysis of compact quantized Riemannian symmetric spaces as the zonal spherical functions on quantum spheres $[\mathrm{NM}, \mathrm{Su}]$. The integer-parameter $q$-ultraspherical polynomials $C_{n}^{N+1}(\cosh (\alpha x) ; q), N \in \mathbb{N}$ studied here correspond in this connection to the zonal spherical functions on a quantization of the real $2 N+3-$ dimensional sphere $S^{2 N+3}=S O(2 N+4 ; \mathbb{R}) / S O(2 N+3 ; \mathbb{R})[\mathrm{Su}]$. For generic values of the spectral parameter $y$, the regular wave function $\Phi_{N}(x, y)+\Phi_{N}(-x, y)$ of Proposition 5.1 is expected to admit an analogous interpretation within the context of the harmonic analysis on noncompact quantized Riemannian symmetric spaces. More precisely, the wave function at issue should correspond to the zonal spherical function on a quantization of the real hyperboloid $\mathrm{H}_{+}^{1,2 N+3}=S \mathrm{O}_{0}(2 \mathrm{~N}+$ $3,1 ; \mathbb{R}) / S O(2 N+3 ; \mathbb{R})[\mathrm{Ka}, \mathrm{KMU}, \mathrm{SV}]$. (The symmetric space is realized explicitly as the upper sheet $H_{+}^{1,2 N+3}=\left\{\mathrm{x} \in \mathbb{R}^{2 N+4} \mid x_{1}^{2}-x_{2}^{2}-\cdots-x_{2 N+4}^{2}=1, x_{1}>0\right\}$ of the two-sheeted hyperboloid $H^{1,2 N+3}=\left\{\mathrm{x} \in \mathbb{R}^{2 N+4} \mid x_{1}^{2}-x_{2}^{2}-\cdots-x_{2 N+4}^{2}=\right.$ 1\}.) To support this conjecture, let us check that for $q \rightarrow 1$ we indeed recover the zonal spherical functions on the hyperboloid $H_{+}^{1,2 N+3}$. It is immediate from Eq. (5.1) that after substitution of $x \rightarrow-2 x / \log (q)$ and $y \rightarrow-y$, the function $(q-1)^{N+1} \Phi_{N, q}(x, y)$ tends for $q \rightarrow 1$ to

$$
\begin{aligned}
& \Phi_{N}(x, y)= \\
& \frac{e^{x(y-N-1)} \sum_{m=0}^{N}(-1)^{m}\left(\begin{array}{l}
N \\
m
\end{array}\right) e^{-2 m x} \prod_{j=1}^{m}(y+N+1-j) \prod_{j=m+1}^{N}(y-j)}{\left(1-e^{-2 x}\right)^{2 N+1} \prod_{j=-N}^{N}(y+j)}
\end{aligned}
$$


The corresponding combinatorial representation of Eq. (5.3) passes in the limit over into

$$
\frac{e^{x(y-N-1)} \sum_{J \subset\{1, \ldots, N\}}(-1)^{\Phi_{j \in J} j} e^{-2 x \sum_{j \in J} j} \prod_{j \in J, k \notin J}\left|\frac{j+k}{j-k}\right| \prod_{j \in J}(y+j) \prod_{k \notin J}(y-k)}{\prod_{j=-N}^{N}(y+j) \sum_{J \subset\{1, \ldots, N+1\}}(-1)^{\sum_{j \in J} j} e^{-2 x \sum_{j \in J} j} \prod_{j \in J, k \notin J}\left|\frac{j+k}{j-k}\right|} .
$$

(The change $N-1 \rightarrow N+1$ in the summation of the denominator stems from absorbtion of a factor $\left(1-e^{-2 x}\right)^{2 N+1}$ originating from the first line of Eq. (5.3), cf. the above comments right after that equation.) In terms of Schur functions the $q=1$ degeneration of the wave function reads

$$
\Phi_{N}(x, y)=\frac{e^{x(y-N-1)} \sum_{\lambda}(-1)^{|\lambda| / 2} b_{\lambda}(y) s_{\lambda}\left(1_{N} e^{-x}\right)}{\prod_{j=-N}^{N}(y+j) \sum_{\mu}(-1)^{|\mu| / 2} s_{\mu}\left(1_{N+1} e^{-x}\right)}
$$

with

$$
b_{\lambda}(y)=\left(y+\alpha_{1}\right) \cdots\left(y+\alpha_{r}\right)\left(y-\beta_{1}\right) \cdots\left(y-\beta_{N-r}\right) .
$$

The sums in the numerator and denominator are now over all partitions $\lambda=$ $\left(\lambda_{1}, \ldots, \lambda_{N}\right)$ and $\mu=\left(\mu_{1}, \ldots, \mu_{N+1}\right)$ characterized by a Frobenius symbol of the form $\left(\alpha_{1}, \ldots, \alpha_{r} \mid \alpha_{1}-1, \ldots, \alpha_{r}-1\right)$, with for the numerator $\alpha_{1} \leq N$ (and $r=0, \ldots, N)$ and for the denominator $\alpha_{1} \leq N+1$ (and $r=0, \ldots, N+1$ ). The integers $\beta_{1}, \ldots, \beta_{N-r}$ are again complementing the integers $\alpha_{1}, \ldots, \alpha_{r}$ such that $\left\{\alpha_{1}, \ldots, \alpha_{r}, \beta_{1}, \ldots, \beta_{N-r}\right\}=\{1, \ldots, N\}$ and the vectors $\mathbf{1}_{N}$ and $\mathbf{1}_{N+1}$ denote the $N$ - and $N+1$-dimensional vectors with unit components. The bispectral pair (5.2a), (5.2b) degenerates in the $q \rightarrow 1$ limit to the differential-difference system

$$
\begin{array}{r}
\left(\frac{\mathrm{d}^{2}}{\mathrm{~d} x^{2}}+(2 N+2) \operatorname{coth}(x) \frac{\mathrm{d}}{\mathrm{d} x}+(N+1)^{2}-y^{2}\right) \Phi_{N}(x, y)=0 \\
\left(1+(N+1) y^{-1}\right) \Phi_{N}(x, y+1)+\left(1-(N+1) y^{-1}\right) \Phi_{N}(x, y-1) \\
=\left(e^{x}+e^{-x}\right) \Phi_{N}(x, y)
\end{array}
$$

The second-order differential operator acting on the l.h.s. of Eq. (5.19a) amounts to the radial component of the Laplace-Beltrami operator on the hyperboloid $H_{+}^{1,2 N+3}=S O_{0}(2 N+3,1 ; \mathbb{R}) / S O(2 N+3 ; \mathbb{R})[\mathrm{He} 1, \mathrm{He} 2, \mathrm{HS}]$. The (regular) even solution of the form $\Phi_{N}(x, y)+\Phi_{N}(-x, y)$ represents the (unnormalized) zonal spherical function on the hyperboloid. The combinatorial and Schur-function formulas for these zonal spherical functions based on Eqs. (5.17) and (5.18a), (5.18b) were first given in [DK].

Remark. 5.2. By substituting $q^{2 x} \rightarrow-q^{2 x}$ in the wave function $\Phi_{N, q}(x, y)$ (5.1), one arrives at a $q$-version of the zonal spherical functions on the noncompact pseudoRiemannian symmetric space $H^{2 N+3,1}=S O_{0}(2 N+3,1 ; \mathbb{R}) / S O_{0}(2 N+2,1 ; \mathbb{R})$. (The symmetric space in question is realized explicitely as the one-sheeted hyperboloid $H^{2 N+3,1}=\left\{\mathrm{x} \in \mathbb{R}^{2 N+4} \mid x_{1}^{2}+\cdots+x_{2 N+3}^{2}-x_{2 N+4}^{2}=1\right\}$.) In this situation (i.e. with $q^{2 x} \rightarrow-q^{2 x}$ ) we have that $\Phi_{N, q}(x, y)$ is regular for $x \in \mathbb{R}$, whence the same is true for the even and odd combinations $\Phi_{N, q}^{ \pm}(x, y)=\Phi_{N, q}(x, y) \pm$ $\Phi_{N, q}(-x, y)$. As before, the representations of Eqs. (5.3) and (5.5a), (5.5b) (with 
$q^{2 x} \rightarrow-q^{2 x}$ ) give rise to combinatorial and Schur-function representations for the pseudo-Riemannian $q$-spherical functions $\Phi_{N, q}^{ \pm}(x, y)$. The corresponding bispectral pair satisfied by these functions now becomes (cf. Eqs. (5.2a), (5.2a))

$$
\begin{array}{r}
t^{-1 / 2}\left(\frac{1+q^{x} t}{1+q^{x}}\right) \Phi_{N, q}^{ \pm}(x+1, y)+t^{1 / 2}\left(\frac{1+q^{x} t^{-1}}{1+q^{x}}\right) \Phi_{N, q}^{ \pm}(x-1, y) \\
=\left(q^{y / 2}+q^{-y / 2}\right) \Phi_{N, q}^{ \pm}(x, y) \\
t^{-1 / 2}\left(\frac{1-q^{y} t}{1-q^{y}}\right) \Phi_{N, q}^{ \pm}(x, y+1)-t^{1 / 2}\left(\frac{1-q^{y} t^{-1}}{1-q^{y}}\right) \Phi_{N, q}^{ \pm}(x, y-1) \\
=\left(q^{x / 2}-q^{-x / 2}\right) \Phi_{N, q}^{ \pm}(x, y)
\end{array}
$$

(where $t=q^{N+1}$ ). After the substitution $x \rightarrow-2 x / \log (q), y \rightarrow-y$ we retrieve for $q \rightarrow 1$ combinatorial and Schur-function formulas for the zonal spherical functions on the pseudo-Riemannian hyperboloid $H^{2 N+3,1}=S O_{0}(2 N+3,1 ; \mathbb{R}) / S O_{0}(2 N+$ $2,1 ; \mathbb{R})$. These formulas, which are of a form given by Eqs. (5.17) and (5.18a), (5.18b) with $\exp (-2 x) \rightarrow-\exp (-2 x)$, were previously introduced in [DK]. The corresponding degeneration of the bispectral pair $(5.20 \mathrm{a}),(5.20 \mathrm{~b})$ satisfied by the zonal spherical functions at issue is given explicitely by

$$
\begin{array}{r}
\left(\frac{\mathrm{d}^{2}}{\mathrm{~d} x^{2}}+(2 N+2) \tanh (x) \frac{\mathrm{d}}{\mathrm{d} x}+(N+1)^{2}-y^{2}\right) \Phi_{N}^{ \pm}(x, y)=0 \\
\left(1+(N+1) y^{-1}\right) \Phi_{N}^{ \pm}(x, y+1)-\left(1-(N+1) y^{-1}\right) \Phi_{N}^{ \pm}(x, y-1) \\
=\left(e^{x}-e^{-x}\right) \Phi_{N}^{ \pm}(x, y)
\end{array}
$$

The second-order differential operator on the l.h.s. of the first equation boils again down to the radial component of the Laplace-Beltrami operator (on the hyperboloid $\left.H^{2 N+3,1}\right)[\mathrm{He} 1, \mathrm{He} 2, \mathrm{HS}]$.

\section{ApPendix A. Reflectionless bidiagonal JaCOBI OPERATORS AND the DISCRETE SATO FORMULA}

In this appendix we provide an explicit parametrization of the Jost function of a reflectionless bidiagonal Jacobi operator in terms of the spectral data. This results in a discrete analog of a well-known formula due to Sato for the Jost function of a one-dimensional reflectionless Schrödinger operator [Sa, SS, DKJM, SW, OSTT, DK]. For relevant background material on the inverse scattering theory of Jacobi operators the reader is referred to [CK, CC, Ca1, Ca2, Fla, To, Te1, Te2].

A.1. Spectral data. We consider the following eigenvalue problem for the bidiagonal Jacobi operator $D=a_{n} T+a_{n-1} T^{-1}$ :

$$
a_{n} \psi(n+1, z)+a_{n-1} \psi(n-1, z)=\left(z+z^{-1}\right) \psi(n, z), \quad n \in \mathbb{Z},
$$

where $z$ denotes a (complex) spectral parameter. Here it will be assumed that the coefficients $a_{n}$ are positive and that $a_{n} \rightarrow 1$ rapidly (say exponentially) as $n \rightarrow \pm \infty$. Consequently, the solutions of Eq. (A.1) have an asymptotics of the form $\alpha_{ \pm} z^{n}+\beta_{ \pm} z^{-n}$ for $n \rightarrow \pm \infty$ (where $\alpha_{ \pm}$and $\beta_{ \pm}$denote certain complex constants). In other words, asymptotically the wave functions decompose in a linear combination of the plane waves $z^{n}$ and $z^{-n}$. Starting from a pair of fundamental solutions for the discrete difference equation, one constructs-by taking a suitable linear combination-the special solution with plane-wave asymptotics of the form 
$z^{n}$ for $n \rightarrow+\infty$. This special solution is referred to as the Jost function (of the first kind). One has that

$$
\psi_{\text {jost }}(n, z) \rightarrow \begin{cases}z^{n} & \text { for } n \rightarrow+\infty \\ \alpha(z) z^{n}-\beta\left(z^{-1}\right) z^{-n} & \text { for } n \rightarrow-\infty\end{cases}
$$

It is clear that

$$
\psi_{\text {jost }}(n,-z)=(-1)^{n} \psi_{\text {jost }}(n, z) .
$$

(Indeed, the r.h.s. of Eq. (A.3) satisfies Eq. (A.1) with $z \rightarrow-z$ and has moreover an asymptotics of the form $(-z)^{n}$ for $n \rightarrow+\infty$.) It thus follows that the quantities $\alpha(z)$ and $\beta(z)$-which characterize the asymptotics of the Jost function at minus infinity-must be even in the spectral parameter $z$.

The zeros of $\alpha(z)$, which (with our assumptions on the coefficients) lie inside the punctured open interval $]-1,1[\backslash\{0\}$, correspond to the discrete spectrum of the Jacobi operator. In other words, for these values of the spectral parameter the wave function lies in the Hilbert space $l^{2}(\mathbb{Z})$ (i.e., it is square summable). Our technical assumptions on the coefficients $a_{n}$ furthermore ensure that these zeros are simple and finite in number. Let us denote the zeros by $\pm z_{1}, \ldots, \pm z_{N}$ with $1>z_{1}>z_{2}>\cdots>z_{N}>0$ (they of course occur in even pairs due to the evenness of $\alpha(z))$ and let $\nu_{1}, \ldots, \nu_{N}>0$ be the corresponding normalization constants

$$
\nu_{j}=\left(\sum_{n \in \mathbb{Z}} \psi_{j o s t}^{2}\left(n, \pm z_{j}\right)\right)^{-1}, \quad j=1, \ldots, N .
$$

(The value of the sum on the r.h.s. of (A.4) does not depend on the choice of the sign in view of Eq. (A.3).) The quantities $1>z_{1}>\cdots>z_{N}>0$ and $\nu_{1}, \ldots, \nu_{N}>0$, together with the refiection coefficient $r(z):=\beta(z) / \alpha(z)$, are referred to as the spectral data of the Jacobi operator. When $r(z)=\beta(z) \equiv 0$ the Jacobi operator is said to be reflectionless.

A.2. The discrete Gelfand-Levitan-Marchenko equation. A remarkable result from the inverse scattering theory of Jacobi operators states that it is possible to reconstruct both the operator (i.e. the coefficients $a_{n}$ ) and the Jost function $\psi_{\text {jost }}(n, z)$ completely from the spectral data [CC, Ca2, Fla, To] (see also [CK, Ca1] for related work pertaining to Jacobi operators on the semi-infinite lattice $\mathbb{N}$ ). Specifically, on has that

$$
\begin{aligned}
& a_{n}=\frac{K(n+1, n+1)}{K(n, n)}, \\
& \psi_{\text {jost }}(n, z)=\sum_{m=n}^{\infty} K(n, m) z^{m} \quad(0<|z| \leq 1),
\end{aligned}
$$

where $K(n, m)$ is a kernel of the form

$$
K(n, m)= \begin{cases}\left(1+F(2 n)+\sum_{l=n+1}^{\infty} k(n, l) F(n+l)\right)^{-1 / 2} & \text { for } m=n \\ K(n, n) k(n, m) & \text { for } m>n\end{cases}
$$


Here the kernel $F(n+l)$ is governed by a function $F(m)$ that encodes the dependence on the spectral data

$$
F(m)=\frac{1}{2 \pi i} \oint r(z) z^{m-1} \mathrm{~d} z+\sum_{j=1}^{N} \nu_{j}\left(z_{j}^{m}+\left(-z_{j}\right)^{m}\right)
$$

(where the integration is along the unit circle with positive orientation), and the kernel $k(n, m), m>n$ is determined from $F(m)$ (A.7) as the (unique) solution of the discrete Gelfand-Levitan-Marchenko equation

$$
k(n, m)+F(n+m)+\sum_{l=n+1}^{\infty} k(n, l) F(l+m)=0, \quad m>n .
$$

A.3. Inverse scattering of reflectionless bidiagonal Jacobi operators. For reflectionless Jacobi operators the reconstruction of the coefficients and Jost function can be made completely explicit [CC, Ca2, Fla, To]. The point is that the kernel associated to $F(m)$ (A.7) becomes in the reflectionless situation of finite rank

$$
F(m)=\left(1+(-1)^{m}\right) \sum_{j=1}^{N} \nu_{j} z_{j}^{m}
$$

We can then solve the discrete Gelfand-Levitan-Marchenko equation (A.8) by means of the following finite-rank Ansatz for the kernel $k(n, m)$ :

$$
k(n, m)=\left(1+(-1)^{n+m}\right) \sum_{j=1}^{N} k_{j}(n) z_{j}^{m} .
$$

Indeed, with this Ansatz the reflectionless discrete Gelfand-Levitan-Marchenko equation becomes of the form

$$
\begin{aligned}
(1+ & \left.(-1)^{n+m}\right) \sum_{j=1}^{N}\left(\nu_{j} z_{j}^{n}+k_{j}(n)\right) z_{j}^{m} \\
& +\sum_{i, j=1}^{N}\left(k_{i}(n) \nu_{j} z_{j}^{m} \sum_{l=n+1}^{\infty}\left(z_{i} z_{j}\right)^{l}\left(1+(-1)^{n+l}\right)\left(1+(-1)^{l+m}\right)\right)=0 .
\end{aligned}
$$

Restriction of the sum over $l$ to the values for which $n+l$ is even and evaluation of the resulting geometric series in $\left(z_{i} z_{j}\right)^{2}$ gives rise to the following finite-dimensional linear system for the kernel functions $k_{1}(n), \ldots, k_{N}(n)$

$$
k_{j}(n)+2 \nu_{j} \sum_{i=1}^{N} k_{i}(n) \frac{z_{i}^{n+2} z_{j}^{n+2}}{1-z_{i}^{2} z_{j}^{2}}=-\nu_{j} z_{j}^{n}, \quad j=1, \ldots, N
$$

This linear system is readily solved with the aid of Cramer's rule:

$$
k_{j}(n)=\frac{\operatorname{det} \mathbf{A}^{(j)}(n)}{\operatorname{det} \mathbf{A}(n)}, \quad j=1, \ldots, N
$$


where $\mathbf{A}(n)$ denotes the $N \times N$ matrix

$$
\mathbf{A}(n)=\left[\begin{array}{ccccc}
1+2 \nu_{1} \frac{z_{1}^{2 n+4}}{1-z_{1}^{4}} & \cdots & 2 \nu_{1} \frac{z_{1}^{n+2} z_{j}^{n+2}}{1-z_{1}^{2} z_{j}^{2}} & \cdots & 2 \nu_{1} \frac{z_{1}^{n+2} z_{N}^{n+2}}{1-z_{1}^{2} z_{N}^{2}} \\
\vdots & \ddots & \vdots & & \vdots \\
2 \nu_{j} \frac{z_{j}^{n+2} z_{1}^{n+2}}{1-z_{j}^{2} z_{1}^{2}} & \cdots & 1+2 \nu_{j} \frac{z_{j}^{2 n+4}}{1-z_{j}^{4}} & \cdots & 2 \nu_{j} \frac{z_{j}^{n+2} z_{N}^{n+2}}{1-z_{j}^{2} z_{N}^{2}} \\
\vdots & & \vdots & \ddots & \vdots \\
2 \nu_{N} \frac{z_{N}^{n+2} z_{1}^{n+2}}{1-z_{N}^{2} z_{1}^{2}} & \cdots & 2 \nu_{N} \frac{z_{N}^{n+2} z_{j}^{n+2}}{1-z_{N}^{2} z_{j}^{2}} & \cdots & 1+2 \nu_{N} \frac{z_{N}^{2 n+4}}{1-z_{N}^{4}}
\end{array}\right]
$$

and $\mathbf{A}^{(j)}(n)$ represents the matrix obtained from $\mathbf{A}(n)$ by replacing the $j^{\text {th }}$ column with (the transpose of) the vector $\left(-\nu_{1} z_{1}^{n}, \ldots,-\nu_{N} z_{N}^{n}\right)$

$$
\mathbf{A}^{(j)}(n)=\left[\begin{array}{ccccc}
1+2 \nu_{1} \frac{z_{1}^{2 n+4}}{1-z_{1}^{4}} & \cdots & -\nu_{1} z_{1}^{n} & \cdots & 2 \nu_{1} \frac{z_{1}^{n+2} z_{N}^{n+2}}{1-z_{1}^{2} z_{N}^{2}} \\
\vdots & \ddots & \vdots & & \vdots \\
2 \nu_{j} \frac{z_{j}^{n+2} z_{1}^{n+2}}{1-z_{j}^{2} z_{1}^{2}} & \cdots & -\nu_{j} z_{j}^{n} & \cdots & 2 \nu_{j} \frac{z_{j}^{n+2} z_{N}^{n+2}}{1-z_{j}^{2} z_{N}^{2}} \\
\vdots & & \vdots & \ddots & \vdots \\
2 \nu_{N} \frac{z_{N}^{n+2} z_{1}^{n+2}}{1-z_{N}^{2} z_{1}^{2}} & \cdots & -\nu_{N} z_{N}^{n} & \cdots & 1+2 \nu_{N} \frac{z_{N}^{2 n+4}}{1-z_{N}^{4}}
\end{array}\right] .
$$

To recover the coefficients $a_{n}$ (A.5a) it suffices to compute the diagonal of the kernel $K(n, m)$ (A.6). We obtain

$$
\begin{aligned}
K^{-2}(n, n) \stackrel{E q s .(\mathrm{A} .9),(\mathrm{A} .10)}{=} & 1+2 \sum_{j=1}^{N} \nu_{j} z_{j}^{2 n}+4 \sum_{i, j=1}^{N} k_{i}(n) \nu_{j} \frac{z_{i}^{n+2} z_{j}^{2 n+2}}{1-z_{i}^{2} z_{j}^{2}} \\
\stackrel{E q .(\text { A.11) }}{=} & 1-2 \sum_{j=1}^{N} k_{j}(n) z_{j}^{n} \\
& \stackrel{\operatorname{Eq.(A.12)} .}{=} \quad \frac{\operatorname{det} \mathbf{A}(n)-2 \sum_{j=1}^{N} z_{j}^{n} \operatorname{det} \mathbf{A}^{(j)}(n)}{\operatorname{det} \mathbf{A}(n)} \\
= & \operatorname{det} \mathbf{A}(n-2) / \operatorname{det} \mathbf{A}(n) .
\end{aligned}
$$

(In the last step one uses that the difference $\mathbf{A}(n-2)-\mathbf{A}(n)$ is a rank-one matrix of the form $\left[2 n_{i} z_{i}^{n} z_{j}^{n}\right]_{1 \leq i, j \leq N}$, whence $\operatorname{det} \mathbf{A}(n-2)-\operatorname{det} \mathbf{A}(n)=-2 \sum_{j=1}^{N} z_{j}^{n} \operatorname{det} \mathbf{A}^{(j)}(n)$. This entails the following determinantal representation for the coefficients $a_{n}$ (A.5a):

$$
a_{n}=\sqrt{\frac{\operatorname{det} \mathbf{A}(n+1) \operatorname{det} \mathbf{A}(n-2)}{\operatorname{det} \mathbf{A}(n) \operatorname{det} \mathbf{A}(n-1)}} .
$$

The above method for deriving the determinantal formula (A.16) for the coefficients $a_{n}$ is patterned after Flaschka [Fla, To]. We will now complement the 
reconstruction of the coefficients with an analogous derivation leading to a determinantal formula for the corresponding Jost function $\psi_{j o s t}(n, z)(\mathrm{A} .5 \mathrm{~b})$. We obtain

$$
\begin{aligned}
\text { (A.17) } \psi_{j o s t}(n, z) & \stackrel{E q .(\mathrm{A} .10)}{=} \quad K(n, n)\left(z^{n}+\sum_{j=1}^{N} k_{j}(n) \sum_{l=n+1}^{\infty}\left(z_{j} z\right)^{l}\left(1+(-1)^{n+l}\right)\right) \\
& =\quad K(n, n)\left(z^{n}+2 \sum_{j=1}^{N} k_{j}(n) \frac{\left(z_{j} z\right)^{n+2}}{1-\left(z_{j} z\right)^{2}}\right) \\
E q s .(\mathrm{A} .12),(\mathrm{A} .15) & \frac{z^{n}\left(\operatorname{det} \mathbf{A}(n)+2 \sum_{j=1}^{N} z_{j}^{n} \operatorname{det} \mathbf{A}^{(j)}(n) \frac{\left(z_{j} z\right)^{2}}{1-\left(z_{j} z\right)^{2}}\right)}{\sqrt{\operatorname{det} \mathbf{A}(n) \operatorname{det} \mathbf{A}(n-2)}} \\
& =\frac{z^{n} \operatorname{det} \mathbf{B}(n, z)}{\sqrt{\operatorname{det} \mathbf{A}(n) \operatorname{det} \mathbf{A}(n-2)}},
\end{aligned}
$$

where $\mathbf{B}(n, z)$ denotes the matrix obtained from $\mathbf{A}(n)$ (A.13) via the substitution $\nu_{j} \rightarrow \nu_{j}\left(1-z^{2} z_{j}^{-2}\right) /\left(1-z^{2} z_{j}^{2}\right)$ :

$$
\mathbf{B}(n, z)=\left[\begin{array}{ccc}
1+2 \nu_{1} \frac{z_{1}^{2 n+4}}{1-z_{1}^{4}}\left(\frac{1-z^{2} z_{1}^{-2}}{1-z^{2} z_{1}^{2}}\right) & \cdots & 2 \nu_{1} \frac{z_{1}^{n+2} z_{N}^{n+2}}{1-z_{1}^{2} z_{N}^{2}}\left(\frac{1-z^{2} z_{1}^{-2}}{1-z^{2} z_{1}^{2}}\right) \\
\vdots & \ddots & \vdots \\
2 \nu_{N} \frac{z_{N}^{n+2} z_{1}^{n+2}}{1-z_{N}^{2} z_{1}^{2}}\left(\frac{1-z^{2} z_{N}^{-2}}{1-z^{2} z_{N}^{2}}\right) & \cdots & 1+2 \nu_{N} \frac{z_{N}^{2 n+4}}{1-z_{N}^{4}}\left(\frac{1-z^{2} z_{N}^{-2}}{1-z^{2} z_{N}^{2}}\right)
\end{array}\right] .
$$

To infer that the expressions on the third and fourth line of Eq. (A.17) are indeed equal, it is convenient to employ the following pole expansion for $\operatorname{det} \mathbf{B}(n, z)$

$$
\operatorname{det} \mathbf{B}(n, z)=\operatorname{det} \mathbf{B}_{0}(n)+\sum_{j=1}^{N} \operatorname{det} \mathbf{B}_{j}(n) \frac{z^{2} z_{j}^{2}}{1-z^{2} z_{j}^{2}},
$$

where $\mathbf{B}_{0}(n)=\lim _{z \rightarrow 0} \mathbf{B}(n, z)=\mathbf{A}(n)$ and $\mathbf{B}_{j}(n)$ is the matrix obtained from $\mathbf{B}(n, z)$ via the substitution $z=z_{j}^{-1}$ after having multiplied the $j^{\text {th }}$ row by a factor $1-z_{j}^{2} z^{2}$ to compensate the singularity. If one first subtracts $z_{k}^{n+2} / z_{j}^{n+2}$ times the $j^{t h}$ column of $\mathbf{B}_{j}(n)$ from its $k^{\text {th }}$ column (for $k=1, \ldots, N, k \neq j$ ) and next multiplies the resulting matrix from the left by the $\operatorname{diag}\left(1-z_{1}^{2} / z_{j}^{2}, \ldots, 1, \ldots, 1-z_{N}^{2} / z_{j}^{2}\right)$ and from the right by $\operatorname{diag}\left(1 /\left(1-z_{1}^{2} / z_{j}^{2}\right), \ldots, 1, \ldots, 1 /\left(1-z_{N}^{2} / z_{j}^{2}\right)\right.$ ) (where the units are in the $j^{\text {th }}$ slot), then one obtains a matrix that equals the matrix $\mathbf{A}^{(j)}(n)$ with its $j^{t h}$ column multiplied by $2 z_{j}^{n}$. Hence, it follows that the expressions on the third and fourth line of Eq. (A.17) coincide.

A.4. The discrete Sato formula. It is possible to evaluate the determinants appearing in the above determinantal representations for the coefficients $a_{n}$ and the Jost function $\psi_{\text {jost }}(n, z)$ (cf. Eqs. (A.16) and (A.17)) in a fully closed form. This evaluation hinges on the Cauchy determinant formula (see e.g. [Ma, p. 67])

$$
\operatorname{det}\left[\frac{1}{1-x_{j} y_{k}}\right]_{1 \leq j, k \leq N}=\frac{\prod_{1 \leq j<k \leq N}\left(x_{j}-x_{k}\right)\left(y_{j}-y_{k}\right)}{\prod_{1 \leq j, k \leq N}\left(1-x_{j} y_{k}\right)} .
$$

To this end we note that $\mathbf{A}(n)$ (A.13) has the structure of an identity matrix plus a matrix of the form NDCD with $\mathbf{N}=\operatorname{diag}\left(\nu_{1}, \ldots, \nu_{N}\right), \mathbf{D}=\operatorname{diag}\left(z_{1}^{n+2}, \ldots, z_{N}^{n+2}\right)$ and $\mathbf{C}=\left[\left(1-z_{j}^{2} z_{k}^{2}\right)^{-1}\right]_{1 \leq j, k \leq N}$. Hence, the determinant of $\mathbf{A}(n)$ boils down to the sum of all principal minors of the matrix NDCD. It is not difficult to evaluate these minors by means of the Cauchy determinant formula in (A.20). (Notice 
that the specialization $x_{j}=y_{j}=z_{j}^{2}, j=1, \ldots, N$ in the Cauchy matrix $[(1-$ $\left.\left.x_{j} y_{k}\right)^{-1}\right]_{1 \leq j, k \leq N}$ reproduces our matrix C.) Having evaluated the determinant of $\mathbf{A}(n)$, the evaluation of the determinant of the matrix $\mathbf{B}(n, z)$ (A.18) follows in turn upon substituting $\nu_{j} \rightarrow \nu_{j}\left(1-z^{2} z_{j}^{-2}\right) /\left(1-z^{2} z_{j}^{2}\right), j=1, \ldots, N$. This way we obtain

$$
\operatorname{det} \mathbf{A}(n)=\tau(n+1), \quad \operatorname{det} \mathbf{B}(n, z)=\chi(n, z)
$$

with

$$
\begin{aligned}
\tau(n) & =\sum_{J \in\{1, \ldots, N\}} \prod_{j \in J} \frac{2 \nu_{j} z_{j}^{2 n+2}}{1-z_{j}^{4}} \prod_{\substack{j, k \in J \\
j<k}}\left(\frac{z_{j}^{2}-z_{k}^{2}}{1-z_{j}^{2} z_{k}^{2}}\right)^{2} \\
\chi(n, z) & =\sum_{J \in\{1, \ldots, N\}} \prod_{j \in J} \frac{2 \nu_{j} z_{j}^{2 n+2}}{1-z_{j}^{4}}\left(\frac{z_{j}^{2}-z^{2}}{1-z_{j}^{2} z^{2}}\right) \prod_{\substack{j, k \in J \\
j<k}}\left(\frac{z_{j}^{2}-z_{k}^{2}}{1-z_{j}^{2} z_{k}^{2}}\right)^{2} .
\end{aligned}
$$

Plugging of the evaluation formulas of Eqs. (A.21), (A.22a), (A.22b) into the determinantal expressions for $a_{n}$ and $\psi_{j o s t}(n, z)$ from Eqs. (A.16) and (A.17), gives rise to the following explicit parametrization of the reflectionless bidiagonal Jacobi operators and their Jost functions in terms of the spectral data.

Proposition A.1 (Spectral parametrization). Let $D=a_{n} T+a_{n-1} T^{-1}$ be a reflectionless bidiagonal Jacobi operator in $l^{2}(\mathbb{Z})$ characterized by the spectral data. $1>z_{1}>\cdots>z_{N}>0$ (corresponding to the discrete spectrum ${ }^{*}$ ) and $\nu_{1}, \ldots, \nu_{N}>0$ (corresponding to the associated normalization constants, cf. Eq. (A.4)). Furthermore, let $\psi_{\text {jost }}(n, z)$ (with $z$ in the punctured unit disc $0<|z| \leq 1$ ) be the (Jost) solution of the eigenvalue equation $D \psi=\left(z+z^{-1}\right) \psi$ characterized by an asymptotics of the form $\psi_{\text {jost }}(n, z) \rightarrow z^{n}$ for $n \rightarrow+\infty$. Then the coefficients and the Jost function are of the form

and

$$
a_{n}=\sqrt{\frac{\tau(n+2) \tau(n-1)}{\tau(n+1) \tau(n)}}
$$

$$
\psi_{\text {jost }}(n, z)=\frac{z^{n} \chi(n, z)}{\sqrt{\tau(n+1) \tau(n-1)}}
$$

where $\tau(n)$ and $\chi(n, z)$ are given by Eqs. (A.22a) and (A.22b), respectively.

As an immediate consequence of Proposition A.1 we are lead to a Sato-type formula for discrete difference operators.

Corollary A.2 (Discrete Sato formula). Let $\tau(n)$ and $\chi(n, z)$ be given by Eqs. (A.22a) and (A.22b) with $0<|z| \leq 1,1>z_{1}>\cdots>z_{N}>0$ and $\nu_{1}, \ldots, \nu_{N}>0$. Then the function

$$
\psi_{j o s t}(n, z)=\frac{z^{n} \chi(n, z)}{\sqrt{\tau(n+1) \tau(n-1)}}
$$

solves the discrete difference equation

$$
a_{n} \psi(n+1, z)+a_{n-1} \psi(n-1, z)=\left(z+z^{-1}\right) \psi(n, z) \quad(n \in \mathbb{Z}),
$$

${ }^{*}$ That is to say, the discrete spectrum consists of $2 N$ eigenvalues of the form $\pm\left(z_{j}+z_{j}^{-1}\right)$, $j=1, \ldots, N$. 
with coefficients given by

$$
a_{n}=\sqrt{\frac{\tau(n+2) \tau(n-1)}{\tau(n+1) \tau(n)}}
$$

Remark A.1. Throughout this appendix we have restricted the spectral parameter $z$ to the punctured unit disc $0<|z| \leq 1$. This guarantees that the seriesrepresentation for the Jost function given by Eq. (A.5b) converges absolutely. We have seen that the spectral values $\pm z_{1}, \ldots, \pm z_{N}$ inside the the punctured interval $]-1,1[\backslash\{0\}$ constitute the discrete spectrum of our Jacobi operator. The values of $z$ on the unit circle correspond to the continuous spectrum. It is clear by analyticity that the statements of Corollary A.2 actually remain valid for all $z \in \mathbb{C} \backslash\left\{0, \pm z_{1}^{-1}, \ldots, \pm z_{N}^{-1}\right\}$. That is to say, the discrete Sato wave function $\psi_{\text {jost }}(n, z)$ of Corollary A.2 solves the corresponding discrete difference equation for all values of the spectral parameter outside the singular points $z=0, \pm z_{j}^{-1}$, $j=1, \ldots, N$. In a similar vein one may also extend the parameter regimes for $z_{j}$ and $\nu_{j}$ in Corollary A.2. More precisely, when substituting the discrete Sato wave function $\psi_{j o s t}(n, z)$ of the corollary in the corresponding discrete difference equation, then we arrive at an equality that holds as an identity between analytic functions (possibly with singularities) in the parameters $z_{j}$ and $\nu_{j}$.

Remark A.2. By definition the parameters $\nu_{j}$ stand for the normalization constants of the Jost function $\psi_{j o s t}(n, z)$ at the discrete spectral values $z_{j}, j=1, \ldots, N$ (cf. Eq. (A.4)). Hence, we have the following nontrivial summation identities for the wave function of Proposition A.1/Corollary A.2

$$
\sum_{n \in \mathbb{Z}} \psi_{j o s t}^{2}\left(n, z_{j}\right)=1 / \nu_{j}, \quad j=1, \ldots, N
$$

In fact, by means of the translation $n \rightarrow n+x$ (with $x \in \mathbb{R}$ ) we can slightly generalize these sums to summation identities of the type

$$
\sum_{n \in \mathbb{Z}} \psi_{j o s t}^{2}\left(n+x, z_{j}\right)=1 / \nu_{j}, \quad j=1, \ldots, N .
$$

Indeed, it is immediate from the explicit expression for the Jost function given by Proposition A.1 that the translation $n \rightarrow n+x$ is equivalent to multiplication of $\psi_{j o s t}(n, z)$ by $z^{x}$ and a reparametrization of the normalization constants $\nu_{j} \rightarrow$ $\nu_{j} z_{j}^{2 x}, j=1, \ldots, N$.

Remark A.3. From the formula of Corollary A.2 one immediately reads-off that the reflectionless Jost function $\psi_{\text {jost }}(n, x)$ has an asymptotics of the form given by Eq. (A.2) with $\alpha(z)=\prod_{j=1}^{N}\left(\frac{1-z^{2} z_{j}^{-2}}{1-z^{2} z_{j}^{2}}\right)$ (and $\left.\beta(z)=0\right)$.

\section{REFERENCES}

Ca1. K.M. Case: On discrete inverse scattering problems. II. J. Math. Phys. 14, 916-920 (1973)

Ca2. K.M. Case: The discrete inverse scattering problem in one dimension. J. Math. Phys. 15, 143-146 (1974)

CC. K.M. Case and S.C. Chui: The discrete version of the Marchenko equation in the inverse scattering problem. J. Math. Phys. 14, 1643-1650 (1973)

CK. K.M. Case and M. Kac: A discrete version of the inverse scattering problem. J. Math. Phys. 14, 594-603 (1973) 
DKJM. E. Date, M. Kashiwara, M. Jimbo, and T. Miwa: Transformation groups for soliton equations. In: M. Jimbo and T. Miwa (eds.), Nonlinear Integrable Systems-Classical Theory and Quantum Theory, World Scientific, Singapore, 1983, pp. 39-119.

DK. J.F. van Diejen and A.N. Kirillov: A combinatorial formula for the associated Legendre functions of integer degree. Adv. Math. (to appear)

DG. J. Duistermaat and F.A. Grünbaum: Differential equations in the spectral parameter. Commun. Math. Phys. 103, 177-240 (1986)

Fla. H. Flaschka: On the Toda lattice. II. Prog. Theor. Phys. 51, 703-716 (1974)

Flu. S. Flügge: Practical Quantum Mechanics I. Springer-Verlag, Berlin, 1971.

GR. G. Gasper and M. Rahman: Basic Hypergeometric Series. Cambridge University Press, Cambridge, 1990.

Gr. F.A. Grünbaum: Some bispectral musings. In: J. Harnad and A. Kasman (eds.), The Bispectral Problem, CRM Proceedings and Lecture Notes vol. 14, Amer. Math. Soc., Providence, R.I., 1998, pp. 31-46.

GH. F.A. Grünbaum and L. Haine: Some functions that generalize the Askey-Wilson polynomials. Commun. Math. Phys. 184, 173-202 (1997)

HS. G.J. Heckman and H. Schlichtkrull: Harmonic Analysis and Special Functions on Symmetric Spaces. Perspect. Math. 16, Academic Press, San Diego, 1994.

He1. S. Helgason: Groups and Geometric Analysis. Academic Press, Orlando, 1984.

He2. S. Helgason: Geometric Analysis on Symmetric Spaces. Amer. Math. Soc., Providence, R.I., 1994 .

Ka. T. Kakehi: Eigenfunction expansion associated with the Casimir operator on the quantum group $\mathrm{SU}_{q}(1,1)$. Duke Math. J. 80, 535-573 (1995)

KMU. T. Kakehi, T. Masuda, and K. Ueno: Spectral analysis of a $q$-difference operator which arises from the quantum $\mathrm{SU}(1,1)$ group. J. Oper. Theory 33, 159-196 (1995)

Ko. B. Kostant: The principal three-dimensional subgroup and the Betti numbers of a complex simple Lie group. Amer. J. Math. 81, 973-1032 (1959)

Ma. I.G. Macdonald: Symmetric Functions and Hall Polynomials (2nd edition). Oxford University Press, Oxford, 1995.

MS. V.B. Matveev and M.A. Salle: Darboux Transformations and Solitons. Springer-Verlag, Berlin, 1991.

NM. M. Noumi and K. Mimachi: Roger's $q$-ultraspherical polynomials on a quantum 2-sphere. Duke Math. J. 63, 65-80 (1991)

OSTT. Y. Ohta, J. Satsuma, D. Takahashi, and T. Tokihiro: An elementary introduction to Sato theory. Prog. Theor. Phys. Suppl. 94, 210-241 (1988)

Ru1. S.N.M. Ruijsenaars: Finite-dimensional soliton systems. In: B. Kupershmidt (ed.), Integrable and Superintegrable Systems. World Scientific, Singapore, 1990, pp. 165-206.

Ru2. S.N.M. Ruijsenaars: Generalized Lamé functions. II. Hyperbolic and trigonometric specializations. J. Math. Phys. (to appear)

Sa. M. Sato: Soliton equations as dynamical systems on an infinite dimensional Grassmann manifolds. RIMS KôkyûRoku No. 439, 30-46 (1981)

SS. M. Sato and Y. Sato: Soliton equations as dynamical systems on infinite dimensional Grassmann manifold. In: H. Fujita, P.D. Lax, and G. Strang (eds.), Nonlinear Partial Differential Equations in Applied Science. Proceedings of the U.S.-Japan Seminar, Tokyo, 1982, Lecture Notes in Num. Appl. Anal. 5, North-Holland Publishing Company, Amsterdam, 1983, pp. 259-271.

SV. Y.S. Soibelman and L. Vaksman: On some problems in the theory of quantum groups. In: A.M. Vershik (ed.), Representation Theory and Dynamical Systems. Adv. Soviet Math. vol. 9, Amer. Math. Soc., Providence, R.I., 1992, pp. 3-55.

SW. G. Segal and G. Wilson: Loop groups and equations of KdV type. Publ. Math. IHES 61, 5-65 (1985)

SZ. V. Spiridonov and A. Zhedanov: Discrete reflectionless potentials, quantum algebras, and orthogonal polynomials. Ann. Phys. (N.Y.) 237, 126-146 (1995)

Su. T. Sugitani: Harmonic analysis on quantum spheres associated with representations of $U_{q}\left(s o_{N}\right)$ and $q$-Jacobi polynomials. Compos. Math. 99, 249-281 (1995)

Te1. G. Teschl: Trace formulas and inverse spectral theory for Jacobi operators. Commun. Math. Phys. 196, 175-202 (1998)

Te2. G. Teschl: Inverse scattering for the Toda hierarchy. Math. Nach. (to appear) 
Ti. E.C. Titchmarsh: Eigenfunction Expansions Associated with Second-order Differential Equations, Part I (2nd edition). Oxford University Press, Oxford, 1962.

To. M. Toda: Theory of Nonlinear Lattices (2nd edition). Springer-Verlag, Berlin, 1989.

Wi. G. Wilson: Bispectral commutative ordinary differential operators. J. reine angew. Math. 442, 177-204 (1993)

Departamento de Matemáticas, Facultad de Ciencias, Universidad de Chile, Casilla 653, Santiago 1 , Chile

Department of Mathematics, HoKkaido University, Sapporo 060-0810, Japan 